\title{
Kalman filter-based Subspace Identification for Operational Modal Analysis under Unmeasured Periodic Excitation
}

\author{
Szymon Greś ${ }^{\mathrm{a}, *}$, Michael Döhler ${ }^{\mathrm{b}}$, Palle Andersen ${ }^{\mathrm{c}}$, Laurent Mevel $^{\mathrm{b}}$ \\ ${ }^{a}$ Aalborg University, Department of Civil and Structural Engineering, Thomas Manns Vej 23, 9000 Aalborg, Denmark \\ ${ }^{b}$ Université Gustave Eiffel, Inria, COSYS/SII, I4S, Campus de Beaulieu, 35042 Rennes, France \\ ${ }^{c}$ Structural Vibration Solutions A/S, NOVI Science Park, 9220 Aalborg, Denmark
}

\begin{abstract}
The modes of linear time invariant mechanical systems can be estimated from output-only vibration measurements under ambient excitation conditions with subspace-based system identification methods. In the presence of additional unmeasured periodic excitation, for example due to rotating machinery, the measurements can be described by a state-space model where the periodic input dynamics appear as a subsystem in addition to the structural system of interest. While subspace identification is still consistent in this case, the periodic input may render the modal parameter estimation difficult, and periodic modes often disturb the estimation of close structural modes. The aim of this work is to develop a subspace identification method for the estimation of the structural parameters while rejecting the influence of the periodic input. In the proposed approach, the periodic information is estimated from the data with a non-steady state Kalman filter, and then removed from the original output signal by an orthogonal projection. Consequently, the parameters of the periodic subsystem are rejected from the estimates, and it is shown that the modes of the structural system are consistently estimated. Furthermore, standard data analysis procedures, like the stabilization diagram, are easier to interpret. The proposed method is validated on Monte Carlo simulations and applied to both a laboratory example and a full-scale structure in operation.
\end{abstract}

Keywords: Operational modal analysis, Ambient excitation, Periodic excitation, Non-steady state Kalman filter, Subspace system identification

\section{Introduction}

The estimation of modal parameters from output-only vibration measurements is the fundamental task of Operational Modal Analysis (OMA). Therein, system identification methods are frequently used to estimate the eigenstructure of a linear system from the accelerations, displacements, velocities or strains recorded on the structure during ambient excitation conditions [1]. Often, the ambient excitation is assumed to be white and stationary, which is sometimes violated, in particular in the presence of periodic movement of rotating machinery on a structure during its operation. Then both ambient and unmeasured periodic forces act on the structure, and the outputs of the corresponding system are described by both the structural system dynamics as well as the dynamics of the periodic excitation. This might render OMA difficult in practice, since the identified eigenstructure then contains a mix of periodic and structural modes [2]. Moreover, when structural and periodic modes are close, the correct identification of the structural parameters may become a problem [3]. The aim of this paper is to develop a subspace identification method for the consistent estimation of the structural modal parameters while rejecting the influence of the unmeasured periodic excitation.

Two classes of methods are commonly used for OMA under periodic excitation. In the first class, the periodic subsignal is separated from the random response signal, and subsequently the modal parameters are estimated with classical methods. For example, time-synchronous-averaging (TSA) is a method extracting periodic waveforms from

${ }^{*}$ Corresponding author; E-mail address: sg@civil.aau.dk 
signals by averaging their blocks synchronized in the angular domain. For OMA, this averaged signal is subtracted from the raw measurements, which results in the removal of the periodic frequencies selected to synchronize the blocks [4]. Angle matching is often achieved with tachometer measurements, which is not practical in real-life applications and was attempted to be overcome in the context of TSA in [5]. A family of methods that does not require tachometer measurements is based on the cepstrum, which is an inverse Fourier transform of the logarithm of spectrum. A number of applications of cepstral lifters to harmonic removal can be found in [6,7]. Although the cepstrum is capable to filter the periodic frequency components out of the spectra of the output data, its empirical premise does not ensure the consistency of the resulting modal parameter estimates. Another group of methods uses a parametric approach to estimate periodic subsignals and removes them from the raw measurements, using for example the Gauss-Newton algorithm [8], or parametric frequency modulation [9].

The second method class comprises techniques that are robust towards intricate input conditions. For example, some methods relax the assumption of random white noise excitation to arbitrary signals, e.g., colored noise, or white noise with periodic components $[10,11]$. In $[2,10-13]$ the authors explore the use of a combination of transmissibility functions under different loading conditions (location or amplitude) to estimate the eigenstructure of the system under white noise excitation mixed with a periodic subsignal. However, the approach proposed therein imposes additional constraints on the system inputs that are not present in classical system identification methods. For example, distinctive excitation conditions are required whose number is known and smaller than the number of output locations [11], which cannot always be ensured in OMA in practice.

A group of classical system identification methods that are well adapted for OMA are stochastic subspace-based methods $[14,15]$. They have been proved to enjoy non-stationary consistency [16], as well as practical aspects like computational efficiency [17] and explicit variance expressions of the identified parameters [18-20] under the white noise assumption. However, when both random and periodic inputs are present, the system output is not strictly ergodic and the output covariances of the system depend on the initial conditions [21, 22]. Since the subspace parameter estimates rely on the output covariances and ergodicity is not given, the consistency of subspace methods is not evident in this setting. In [23] the authors prove consistency of both system and oscillatory parameters for estimates from a covariance-based subspace identification method. This fact can be used to discard the periodic poles of the system based on the consistent estimate of its eigenstructure, which was illustrated on a theoretical example in [24].

The current paper builds upon this work with the aim to develop a robust subspace method in the context of OMA for the identification of structural modes while rejecting the periodic contribution of the signals. The system matrices of the underlying state space model are time-invariant, and the unmeasured periodic excitation is assumed to be a superposition of sinusoids. The proposed approach operates in the data-driven output-only stochastic subspace identification framework. The periodic excitation is modeled as a part of the system states, thus it can be optimally estimated from the data with a non-steady state Kalman filter. A subspace algorithm is proposed, where the raw output data are projected on the orthogonal complement of the estimated periodic sequence in order to remove the latter from the raw data. This is particularly useful when the periodic modes are close to structural modes, or when they are of high energy and then may mask the system response to the random part of the input. Subsequently, the periodic excitation parameters are rejected from the resulting estimates of the system matrices in subspace identification based on the projected signal, and the eigenstructure of the underlying structural system is properly identified.

This paper is organized as follows. The background and modeling of the considered problem is given in Section 2. The proposed method for the removal of the periodic subsignal and subsequent subspace identification is derived in Section 3, including a proof of the consistency of the method. The method is validated on Monte Carlo simulations in Section 4. An application to two experimental cases is reported in Section 5, namely to a plate in the laboratory and to a full-scale ship in operation.

\section{Problem statement}

In this section, the vibration model is recalled, and the impact of the periodic excitation on the models and identified parameters is stated. The latter is illustrated by Monte Carlo simulations of a mass-spring chain system. 


\subsection{Stochastic system models and parameters}

Assume that the vibration behavior of a viscously damped, linear time-invariant (LTI) structural system with $m$ degrees of freedom is described by the differential equation

$$
\mathcal{M} \ddot{q}(t)+C \dot{q}(t)+\mathcal{K} q(t)=\mathbf{f}(t)
$$

where $t$ denotes continuous time, and $\mathcal{M}, C, \mathcal{K} \in \mathbb{R}^{m \times m}$ denote mass, damping and stiffness matrices, respectively. Vectors $q(t) \in \mathbb{R}^{m}$ and $\mathbf{f}(t) \in \mathbb{R}^{m}$ contain the continuous-time displacements and the unmeasured external forces, respectively. Let system (1) be observed by sensors measuring, e.g., accelerations, velocities or displacements, at $r$ degrees of freedom (DOF) of the structure, collected in the vector

$$
y(t)=C_{a} \ddot{q}(t)+C_{v} \dot{q}(t)+C_{d} q(t)+\tilde{v}(t)
$$

where $y(t) \in \mathbb{R}^{r}$ is the output vector, $\tilde{v}(t) \in \mathbb{R}^{r}$ denotes the sensor noise, and matrices $C_{a}, C_{v}, C_{d} \in \mathbb{R}^{r \times m}$ select the respective type of the output at the measurement DOFs.

When defining the states $x(t)=\left[\begin{array}{ll}q(t)^{T} & \dot{q}(t)^{T}\end{array}\right]^{T} \in \mathbb{R}^{2 m}$, the structural system model (1) with output equation (2) yields the continuous-time state space model

$$
\begin{aligned}
& \dot{x}(t)=A_{c}^{\text {sys }} x(t)+w(t), \\
& y(t)=C^{\text {sys }} x(t)+v(t),
\end{aligned}
$$

where the state matrix $A_{c}^{\text {sys }} \in \mathbb{R}^{2 m \times 2 m}$, observation matrix $C^{\text {sys }} \in \mathbb{R}^{r \times 2 m}$, process noise $w(t) \in \mathbb{R}^{2 m}$ and output noise $v(t) \in \mathbb{R}^{r}$ are

$$
A_{c}^{\text {sys }}=\left[\begin{array}{cc}
0 & I \\
-\mathcal{M}^{-1} \mathcal{K} & -\mathcal{M}^{-1} \mathcal{C}
\end{array}\right], \quad C^{\text {sys }}=\left[\begin{array}{ll}
C_{d}-C_{a} \mathcal{M}^{-1} \mathcal{K} & C_{v}-C_{a} \mathcal{M}^{-1} C
\end{array}\right], \quad w(t)=\left[\begin{array}{c}
0 \\
\mathcal{M}^{-1}
\end{array}\right] \mathbf{f}(t),
$$

and $v(t)=C_{a} \mathcal{M}^{-1} \mathbf{f}(t)+\tilde{v}(t)$, where the model order is $n=2 \mathrm{~m}$. When sampled at discrete time instants $t=k \tau$, where $\tau$ is the time step and $k$ is an integer, the resulting discrete-time state space model is given by [25]

$$
\begin{aligned}
x_{k+1} & =A^{\text {sys }} x_{k}+w_{k}, \\
y_{k} & =C^{\text {sys }} x_{k}+v_{k},
\end{aligned}
$$

where $x_{k}=x(k \tau) \in \mathbb{R}^{2 m}$ are the discrete states and $A^{\text {sys }}=\exp \left(A_{c}^{\text {sys }} \tau\right) \in \mathbb{R}^{2 m \times 2 m}$ is the state transition matrix. Note that the index $(\cdot)^{\text {sys }}$ indicates here that the respective parameter refers in particular to the structural system, which is distinguished from the periodic part denoted by $(\cdot)^{\text {per }}$ later on in the paper. The discrete process noise $w_{k}$ and output noise $v_{k}$ are assumed to be zero-mean white noise vectors with finite fourth order moments. For simplicity, assume in addition that they are Gaussian. Their covariance matrix is given by

$$
\mathrm{E}\left(\left[\begin{array}{c}
w_{k} \\
v_{k}
\end{array}\right]\left[\begin{array}{ll}
w_{l} & v_{l}
\end{array}\right]\right)=\left[\begin{array}{cc}
Q & S \\
S^{T} & R
\end{array}\right] \delta_{k l} \geq 0 .
$$

Matrices $A^{\text {sys }}$ and $C^{\text {sys }}$ are of particular interest since they are used to identify the modal parameters of the structure. They can be estimated from data $\left\{y_{k}\right\}$ with subspace identification methods, based on the column space of an adequate projection of the data sequences $[14,20]$. The $i$-th natural frequency $f_{i}^{\text {sys }}$, damping ratio $\zeta_{i}^{\text {sys }}$ and mode shape $\varphi_{i}^{\text {sys }}$ of the underlying structural system are related to the eigenvalue $\lambda_{i}^{\text {sys }}$ and eigenvector $\phi_{i}^{\text {sys }}$ of $A^{\text {sys }}$ by

$$
f_{i}^{\mathrm{sys}}=\frac{\left|\lambda_{c i}^{\mathrm{sys}}\right|}{2 \pi}, \zeta_{i}^{\mathrm{sys}}=\frac{-\mathfrak{R}\left(\lambda_{c i}^{\mathrm{sys}}\right)}{\left|\lambda_{c i}^{\mathrm{sys}}\right|}, \varphi_{i}^{\mathrm{sys}}=C^{\mathrm{sys}} \phi_{i}^{\mathrm{sys}}
$$

where the $i$-th eigenvalue $\lambda_{c i}^{\text {sys }}$ of the continuous-time system yields $\exp \left(\lambda_{c i}^{\mathrm{sys}} \tau\right)=\lambda_{i}^{\mathrm{sys}}$. 


\subsection{Impact of mixed random and periodic excitation on the state-space model}

In this section, the influence of unmeasured periodic excitation on the classical formulation of the state-space model (5)-(6) and its parameters is developed and illustrated on a numerical example.

Assume that a deterministic periodic force $u(t)$ acts on the system in addition to the random noise input $w(t)$. For simplicity of notation let this periodic force be one-dimensional, i.e., $u(t) \in \mathbb{R}$, and let $s_{u} \in \mathbb{R}^{m}$ be an index vector containing 0's and 1's, indicating at which degree(s) of freedom the periodic force acts on the structure. Denote the resulting states as $x_{k}^{\text {sys }}$, containing the displacements and velocities at the DOFs of the structure under both unknown noise and periodic forces. Then, the continuous-time state space model (3)-(4) becomes

$$
\begin{aligned}
\dot{x}^{\text {sys }}(t) & =A_{c}^{\text {sys }} x^{\text {sys }}(t)+\mathbf{b} u(t)+w(t), \\
y(t) & =C^{\text {sys }} x^{\text {sys }}(t)+\mathbf{d} u(t)+v(t),
\end{aligned}
$$

where

$$
\mathbf{b}=\left[\begin{array}{c}
0 \\
\mathcal{M}^{-1}
\end{array}\right] s_{u} \in \mathbb{R}^{2 m}, \mathbf{d}=C_{a} \mathcal{M}^{-1} s_{u} \in \mathbb{R}^{r} .
$$

Since the periodic force $u(t)$ is unmeasured, it is the goal to eliminate it from the state space model and include its effects in the system matrices and in the state vector. Assuming that the periodic force contains $h$ frequency components of the shape

$$
u(t)=\sum_{i=1}^{h} a_{i} \sin \left(\omega_{i} t+g_{i}\right),
$$

where $a_{i}, g_{i}, \omega_{i} \in \mathbb{R}$ are (unknown) amplitude, shift and circular frequencies of the periodic input components, then these components can become part of a combined state vector in order to eliminate the periodic input component in model (8)-(9), as follows. Define

$$
x^{\mathrm{per}}(t)=\left[\begin{array}{c}
a_{1} \sin \left(\omega_{1} t+g_{1}\right) \\
a_{1} \cos \left(\omega_{1} t+g_{1}\right) \\
\vdots \\
a_{h} \sin \left(\omega_{h} t+g_{h}\right) \\
a_{h} \cos \left(\omega_{h} t+g_{h}\right)
\end{array}\right] \in \mathbb{R}^{2 h}, \quad \text { thus } \dot{x}^{\mathrm{per}}(t)=\left[\begin{array}{c}
\omega_{1} a_{1} \cos \left(\omega_{1} t+g_{1}\right) \\
-\omega_{1} a_{1} \sin \left(\omega_{1} t+g_{1}\right) \\
\vdots \\
\omega_{h} a_{h} \cos \left(\omega_{h} t+g_{h}\right) \\
-\omega_{h} a_{h} \sin \left(\omega_{h} t+g_{h}\right)
\end{array}\right] .
$$

The relationship between $\dot{x}^{\text {per }}(t)$ and $x^{\text {per }}(t)$ follows as

$$
\dot{x}^{\text {per }}(t)=A_{c}^{\text {per }} x^{\text {per }}(t), \quad \text { where } A_{c}^{\text {per }}=\operatorname{diag}\left(H_{1}, \ldots, H_{h}\right) \text { and } H_{i}=\left[\begin{array}{cc}
0 & \omega_{i} \\
-\omega_{i} & 0
\end{array}\right],
$$

and the relationship between $u(t)$ and $x^{\mathrm{per}}(t)$ is given by $u(t)=s_{h} x^{\mathrm{per}}(t)$, where $s_{h}=\left[\begin{array}{lllll}1 & 0 & \ldots & 1 & 0\end{array}\right] \in \mathbb{R}^{1 \times 2 h}$. Thus, $\operatorname{defining} A_{c}^{\mathbf{b}}=\mathbf{b} s_{h} \in \mathbb{R}^{2 m \times 2 h}$ and $C^{\mathrm{per}}=\mathbf{d} s_{h} \in \mathbb{R}^{r \times 2 h}$, the state space model (8)-(9) containing the unmeasured periodic input $u(t)$ can be equivalently rewritten as a combined state space model without the periodic input as

$$
\begin{aligned}
{\left[\begin{array}{c}
\dot{x}^{\mathrm{sys}}(t) \\
\dot{x}^{\mathrm{per}}(t)
\end{array}\right] } & =\left[\begin{array}{cc}
A_{c}^{\mathrm{sys}} & A_{c}^{\mathbf{b}} \\
0 & A_{c}^{\mathrm{per}}
\end{array}\right]\left[\begin{array}{l}
x^{\mathrm{sys}}(t) \\
x^{\mathrm{per}}(t)
\end{array}\right]+\left[\begin{array}{c}
w(t) \\
0
\end{array}\right], \\
y(t) & =\left[\begin{array}{ll}
C^{\mathrm{sys}} & C^{\mathrm{per}}
\end{array}\right]\left[\begin{array}{l}
x^{\mathrm{sys}}(t) \\
x^{\mathrm{per}}(t)
\end{array}\right]+v(t),
\end{aligned}
$$

where the model order is $n=2(m+h)$. Note that due to the upper right block structure of the state matrix, the eigenvalues of the combined system are the combined sets of eigenvalues of $A_{c}^{\text {sys }}$ and of $A_{c}^{\text {per }}$. While the eigenvectors of the combined state matrix regarding the structural part become $\left[\phi_{i}^{\text {sys } T} 0\right]^{T}$, the resulting mode shapes are $\varphi_{i}^{\text {sys }}$, as in system (8)-(9).

Sampling model (12)-(13) at discrete time instants $t=k \tau$ yields the combined discrete-time state-space model

$$
\begin{aligned}
{\left[\begin{array}{c}
x_{k+1}^{\text {sys }} \\
x_{k+1}^{\text {per }}
\end{array}\right] } & =\left[\begin{array}{cc}
A^{\text {sys }} & A^{\mathbf{b}} \\
0 & A^{\mathrm{per}}
\end{array}\right]\left[\begin{array}{c}
x_{k}^{\mathrm{sys}} \\
x_{k}^{\mathrm{per}}
\end{array}\right]+\left[\begin{array}{c}
w_{k} \\
0
\end{array}\right] \\
y_{k} & =\left[\begin{array}{ll}
C^{\mathrm{sys}} & C^{\mathrm{per}}
\end{array}\right]\left[\begin{array}{c}
x_{k}^{\mathrm{sys}} \\
x_{k}^{\mathrm{per}}
\end{array}\right]+v_{k},
\end{aligned}
$$


where the combined system matrix yields

$$
\left[\begin{array}{cc}
A^{\text {sys }} & A^{\mathbf{b}} \\
0 & A^{\text {per }}
\end{array}\right]=\exp \left(\left[\begin{array}{cc}
A_{c}^{\text {sys }} & A_{c}^{\mathbf{b}} \\
0 & A_{c}^{\text {per }}
\end{array}\right] \tau\right) .
$$

Recall that the first $2 m$ components of the process noise are related to the ambient excitation of the system, while the deterministic periodic excitation is translated into the states $x_{k}^{\text {per }}$ as shown above.

The eigenvalues of $A^{\text {sys }}$ and $A^{\text {per }}$ are denoted by $\lambda_{i}^{\text {sys }}, \bar{\lambda}_{i}^{\text {sys }}, i=1, \ldots, m$, and $\lambda_{i}^{\text {per }}, \bar{\lambda}_{i}^{\text {per }}, i=1, \ldots, h$, respectively. The eigenvalues of the structural system yield $\left|\lambda_{i}^{\text {sys }}\right|<1$, while the eigenvalues of the periodic part of the system are situated on the unitary circle, i.e., $\left|\lambda_{i}^{\text {per }}\right|=1$. Hence both kinds of modes can be distinguished in the combined state matrix. Moreover, the periodic excitation (10) corresponds to undamped modes of the periodic part of the continuoustime system as can be seen in Equations (11) and (12), i.e., $\mathfrak{R}\left(\lambda_{c i}^{\mathrm{per}}\right)=0$.

These properties are illustrated in the context of a system subjected to mixed random and periodic excitation in the following section.

\subsection{Illustrative example}

Consider a 6 DOF mass-spring chain system that, for any consistent set of units, is modeled with spring stiffness $k_{1}=k_{3}=k_{5}=100$ and $k_{2}=k_{4}=k_{6}=200$, mass of each element $m_{i}=1 / 20$ and a proportional damping matrix such that each mode has a damping ratio of $\zeta_{i}^{\text {sys }}=3 \%$. The system is subjected to white noise excitation in all DOFs and sampled with a frequency of $50 \mathrm{~Hz}$ for 2000 seconds. An additional sinusoidal excitation with a frequency of 8.69 $\mathrm{Hz}$, close to the third natural frequency of the system, is applied at all DOFs. This excitation is devised to mimic a periodic input from, e.g., an engine rotating at a constant speed. The resulting acceleration responses are obtained at DOFs 1, 2 and 5. Gaussian white noise with 5\% of the standard deviation of the output is added to the response at each channel.

The modal parameters of the combined system model including the periodic part are depicted in Table 1. The resulting eigenvalues of the discrete-time system and the respective continuous-time system are shown in the complex plane in Figure 1. It can be seen that the periodic and the system poles can indeed be distinguished in the complex plane. This fact will be used to estimate the periodic states and consequently the periodic subsignal of the output with the method proposed in this paper.

Table 1: Exact modal parameters of the chain system, and properties of the periodic excitation.

\begin{tabular}{|c|c|c|c|c|c|c|c|c|c|c|c|c|c|}
\hline \multicolumn{7}{|c|}{ Natural frequency $(\mathrm{Hz})$} & \multicolumn{7}{|c|}{ Damping ratio $(\%)$} \\
\hline$f_{1}^{\text {sys }}$ & $f_{2}^{\mathrm{sys}}$ & $f_{3}^{\text {sys }}$ & $f_{4}^{\text {sys }}$ & $f_{5}^{\mathrm{sys}}$ & $f_{6}^{\mathrm{sys}}$ & $f_{1}^{\text {per }}$ & $\zeta_{1}^{\text {sys }}$ & $\zeta_{2}^{\text {sys }}$ & $\zeta_{3}^{\text {sys }}$ & $\zeta_{4}^{\text {sys }}$ & $\zeta_{5}^{\text {sys }}$ & $\zeta_{6}^{\text {sys }}$ & $\zeta_{1}^{\text {per }}$ \\
\hline 1.93 & 5.62 & 8.68 & 14.49 & 15.85 & 17.01 & 8.69 & 3 & 3 & 3 & 3 & 3 & 3 & 0 \\
\hline
\end{tabular}
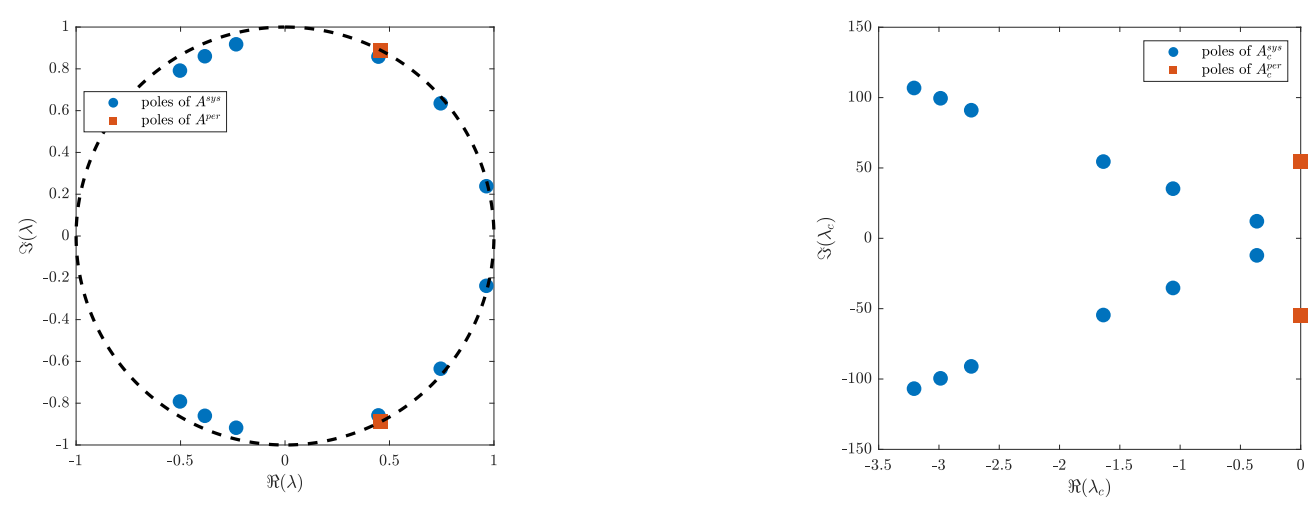

Figure 1: Discrete-time and continues-time poles of the system from (12) and (14) respectively. 

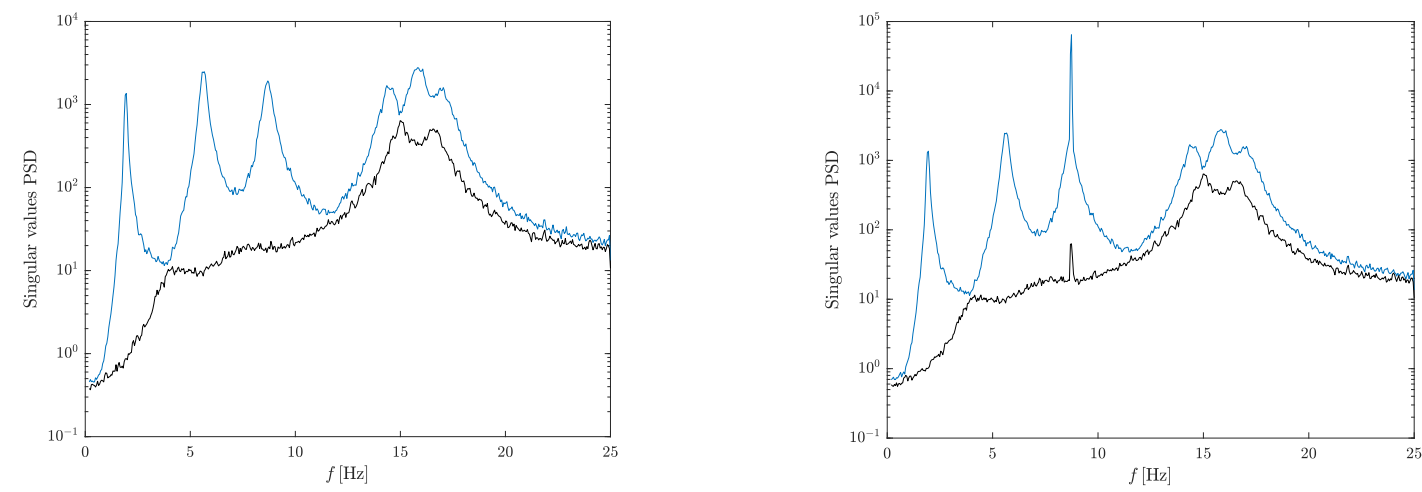

Figure 2: Two largest singular values of PSD matrix from output data of the system subjected to random (left) and mixed random and periodic (right) excitation.
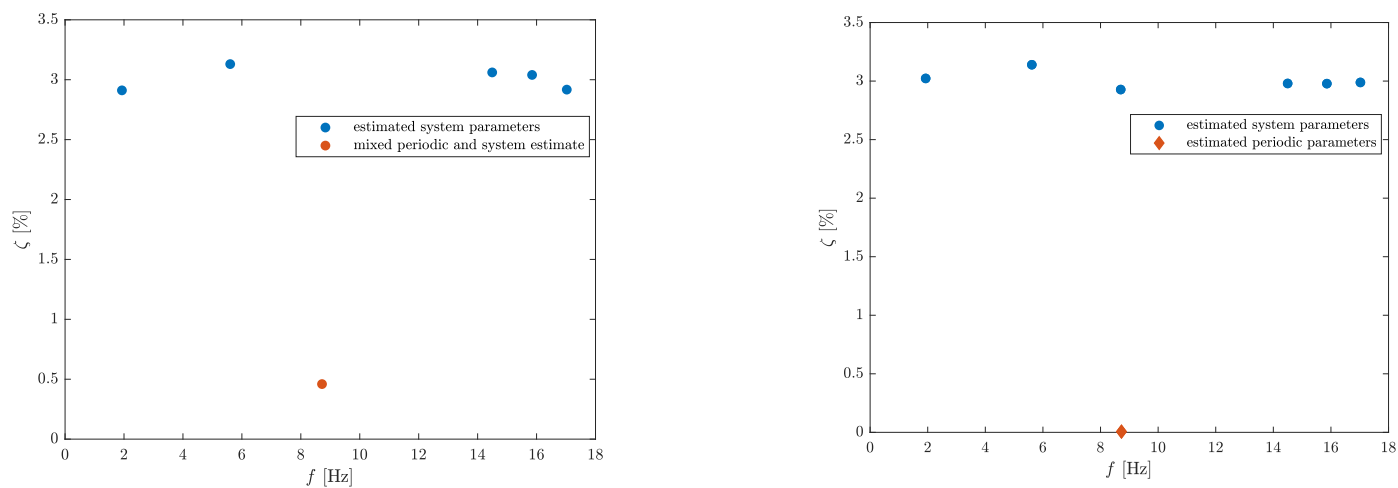

Figure 3: Estimates of natural frequency and damping ratio from one simulation. Model order 12 (left) and 14 (right).

Furthermore, the presence of periodic poles can be seen in the Power Spectral Density (PSD) of the data [26]. Figure 2 shows the two largest singular values of the PSD matrix constructed from the structural responses with and without the periodic excitation. It can be observed that the fundamental frequency of the periodic excitation manifests as a sharp spike in the output power spectra. However, since its frequency is close to a system pole, both poles cannot be easily distinguished visually from the PSD plot.

The next example illustrates the estimation of the natural frequencies and damping ratios by a Monte Carlo experiment with 1000 simulations. The output-only data driven subspace-based system identification with the unweighted principal component (SSI-UPC) $[14,15]$ is deployed, using 30 time lags for the data Hankel matrix and estimating the system matrices at model orders of 12 and 14. For both model orders, the respective sets of modes are tracked in each simulation. The estimates of the natural frequency and damping ratio from one simulation are depicted in Figure 3. Based on all simulations, Figures 4 and 5 show the histograms of the natural frequencies and the damping ratios of the mode closest to the periodic frequency for both model orders of 12 and 14.

Recall that model order 12 corresponds to the structural system, and model order 14 corresponds to the combined system with the periodic mode. When the mode close to the periodic mode is estimated at model order 12, it can be observed in the left parts of Figures 3-5 that its frequency and damping ratio estimates are in between the structural mode (at $8.68 \mathrm{~Hz}$ and 3\% damping) and the periodic mode (at $8.69 \mathrm{~Hz}$ and $0 \%$ damping). When estimating the mode at model order 14, the mean values of the histogram of the natural frequency and the damping ratio in the right parts of Figures 4 and 5 are close to the exact values. This infers that by augmenting the exact model order to account for the periodic pole, both the system and periodic parameters are consistently estimated, which agrees with the state-space model proposed in (14) and (15) in the previous section. This fact is used in the method proposed in the following section. 

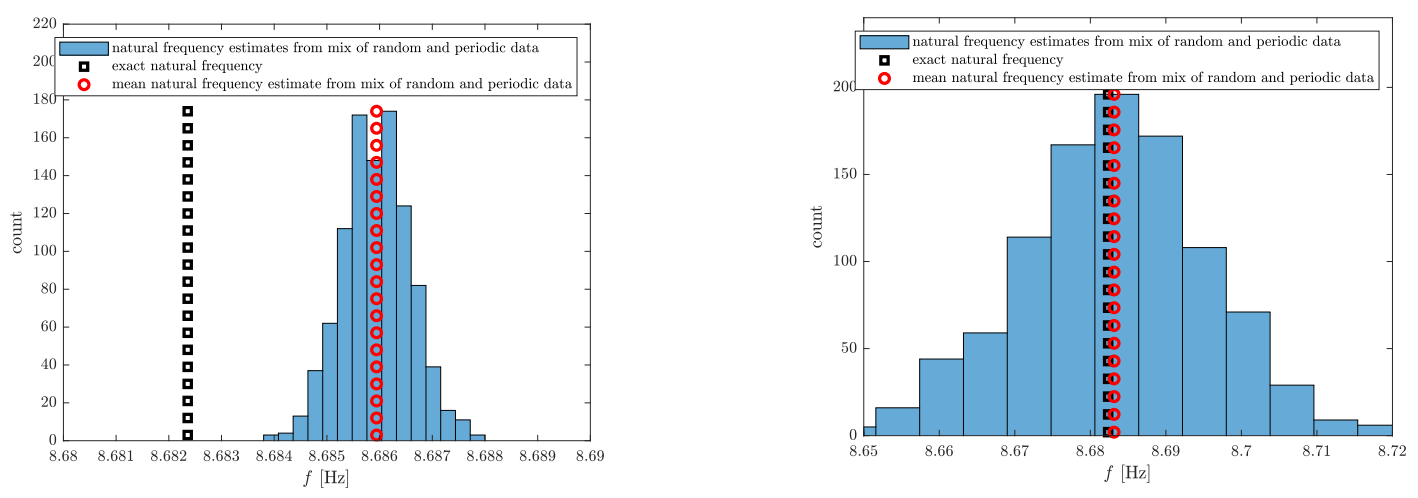

Figure 4: Histograms of the natural frequency of the third mode identified with model order 12 (left) and 14 (right).
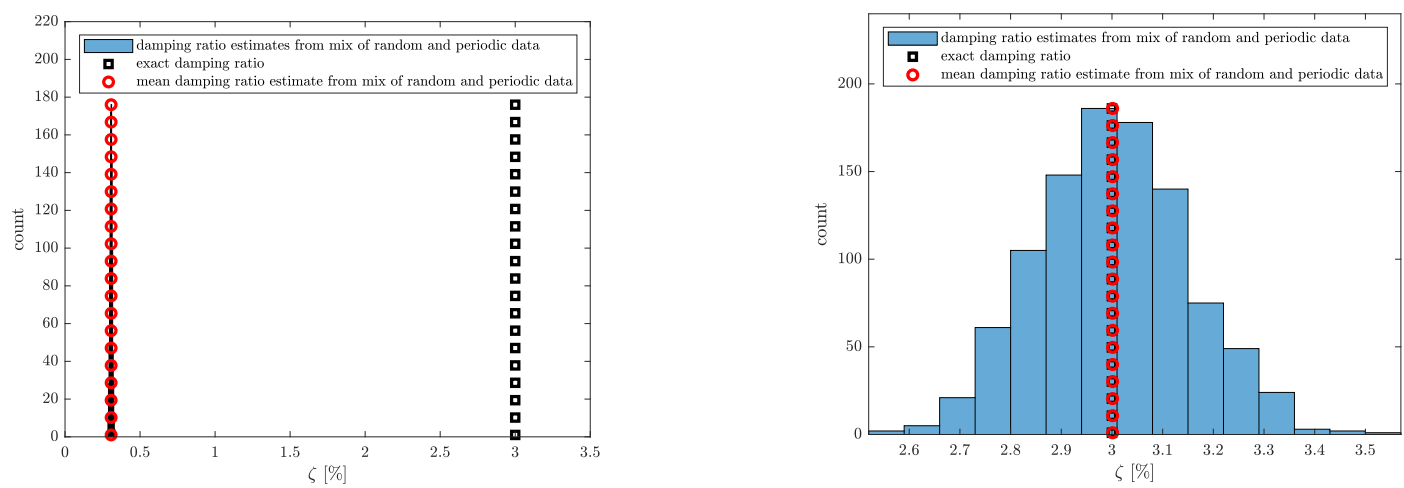

Figure 5: Histograms of the damping ratio of the third mode identified with model order 12 (left) and 14 (right).

\section{Subspace-based system identification under mixed periodic and random excitation}

As shown in the previous section, the exact model order of the structural system can be increased by the number of periodic poles that are present in the data. Using output-only stochastic subspace identification, consistent estimates of both structural and periodic poles can be obtained [23], and the latter can be rejected from the modal estimates as illustrated on the theoretical example in the previous section. However, the exact model order is unknown in practical applications and the periodic excitation may coincide with a natural frequency of the structure, or it may be of high energy that masks the system response to the random part of the input. In these cases, it is desirable to discard the periodic excitation from the data and without additional knowledge of, e.g., tachometer measurements. In this section, a scheme for the removal of the periodic subsignal is proposed, based on three steps:

1. Estimation of the periodic poles by subspace-based system identification from the raw output data,

2. Estimation of the periodic subsignal using the Kalman filter,

3. Projection of the row space of the raw output data onto the orthogonal complement of the row space of the periodic subsignal estimate.

Based on the projected signal, any further signal processing can be carried out for the analysis of the structural system response, where the nuisance from the periodic inputs is removed. In particular, subspace-based system identification can be used to estimate only the structural system modes, which is detailed in the remainder of this section.

In the following, it is assumed that the first step has already been carried out and that the periodic modes are selected, including the modes corresponding to the harmonics, for example based on indicators developed in [27, 28]. 
In practical applications, the selection of periodic poles can be done with simple indicators like kurtosis [27, 28], entropy [29] or damping ratios [30].

\subsection{Estimation of periodic subsignal}

The periodic subsignal is estimated based on the Kalman filter. Note that the eigenvalues of the periodic subsystem are situated on the unitary circle, thus the considered combined system (14)-(15) is not a classical stable system. Moreover, the periodic part does not have process noise. Nevertheless, the Kalman filter can be applied and is stable, as detailed in $[23,31]$.

For the estimation of the periodic subsignal the Kalman filter states are retrieved in the modal basis in order to distinguish the states referring to the periodic poles. For this, the Kalman filter states are obtained first in an arbitrary basis, namely in the basis corresponding to estimates of the system matrices $\{A, C, Q, R, S\}$ of the combined system corresponding to (14)-(15). These system matrices can be estimated from data using for example the SSI-UPC method described in [14]. Then, the Kalman filter states are converted to the modal basis, and the modal states corresponding to the periodic modes are selected to estimate the periodic subsignal. This procedure is detailed in the following.

With the Kalman filter, unbiased and minimum variance estimates $\hat{x}_{k+1}$ of the states $x_{k+1}$ are obtained. In this work, the non-steady state Kalman filter is used that accounts for the correlation between process and output noise, based on [32]. The initial state estimate is assumed to be $\hat{x}_{0}=0 \in \mathbb{R}^{n}$, and the initial error covariance matrix is assumed as $P_{0}=\mathcal{I}_{n} \in \mathbb{R}^{n \times n}$. Then, the recursive filter equations are

$$
\begin{aligned}
K_{k} & =\left(A P_{k} C^{T}+S\right)\left(R+C P_{k} C^{T}\right)^{-1}, \\
\hat{x}_{k+1} & =\left(A-K_{k} C\right) \hat{x}_{k}+K_{k} y_{k}, \\
P_{k+1} & =A P_{k} A^{T}+Q-K_{k}\left(A P_{k} C^{T}+S\right)^{T},
\end{aligned}
$$

where $K_{k} \in \mathbb{R}^{n \times r}$ is the gain matrix, which converges to the steady state gain $K \in \mathbb{R}^{n \times r}$ for increasing $k$. Consequently, defining the innovations $e_{k}=y_{k}-C \hat{x}_{k} \in \mathbb{R}^{r}$, the Kalman filter states and system outputs yield

$$
\begin{aligned}
\hat{x}_{k+1} & =A \hat{x}_{k}+K_{k} e_{k}, \\
y_{k} & =C \hat{x}_{k}+e_{k},
\end{aligned}
$$

which is the state-space model in innovation form. The states are not unique since for any invertible matrix $V \in \mathbb{R}^{n \times n}$ the linear transformations

$$
\hat{x}_{k}^{V}=V^{-1} \hat{x}_{k}, A^{V}=V^{-1} A V, \quad C^{V}=C V, \quad K_{k}^{V}=V^{-1} K_{k}
$$

yield the state-space model

$$
\begin{aligned}
\hat{x}_{k+1}^{V} & =A^{V} \hat{x}_{k}^{V}+K_{k}^{V} e_{k}, \\
y_{k} & =C^{V} \hat{x}_{k}^{V}+e_{k}
\end{aligned}
$$

that is equivalent in terms of outputs, eigenvalues and mode shapes. Without loss of generality, the modal basis is chosen for the transformation in order to distinguish the states that are corresponding to the periodic part of the system. More precisely, matrix $V$ is chosen based on the eigenvectors of $A$ as follows. Recall that $\phi_{i}^{\text {sys }}$ and $\bar{\phi}_{i}^{\text {sys }}, i=1, \ldots, m$ and $\phi_{i}^{\text {per }}$ and $\bar{\phi}_{i}^{\text {per }}, i=1, \ldots, h$, are the pairs of conjugated complex eigenvectors of $A$ corresponding to the structural system and to the periodic part, respectively. Analogously, $\lambda_{i}^{\text {sys }}$ and $\bar{\lambda}_{i}^{\text {sys }}, i=1, \ldots, m$ and $\lambda_{i}^{\text {per }}$ and $\bar{\lambda}_{i}^{\text {per }}, i=1, \ldots, h$, are the respective eigenvalues. Applying the transformation defined by

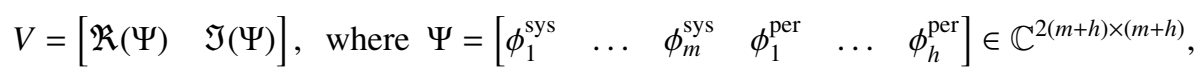

yields the state-space model with real-valued system matrices in the modal basis with

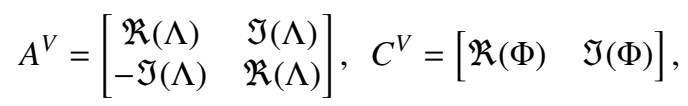

where $\Lambda=\operatorname{diag}\left(\lambda_{1}^{\mathrm{sys}}, \ldots, \lambda_{m}^{\mathrm{sys}}, \lambda_{1}^{\mathrm{per}}, \ldots, \lambda_{h}^{\mathrm{per}}\right)$ and $\Phi=C \Psi=\left[\varphi_{1}^{\mathrm{sys}} \ldots \varphi_{m}^{\mathrm{sys}} \varphi_{1}^{\mathrm{per}} \ldots \varphi_{h}^{\mathrm{per}}\right]$ contain one element of each complex conjugated pair of eigenvalues and mode shapes, respectively. 
Note that it is impossible to obtain the system matrices and the Kalman filter states in the same state basis as in (14)-(15) when estimated from data. However, the previous transformation of the system matrices into the modal basis $\left\{A^{V}, C^{V}, K_{k}^{V}\right\}$ yields a canonical format when the system matrices are identified in an arbitrary basis. Moreover, the states corresponding to the system and to the periodic parts are decoupled in the state vector $\hat{x}_{k}^{V}$. Subsequently, the states corresponding to the periodic part can be selected from the respective entries of $\hat{x}_{k}^{V}$ and the periodic output subsignal related to these states estimated. For this, define a selection matrix $S$ with

$$
S=\left[\begin{array}{cccc}
0_{m \times m} & & & \\
& \mathcal{I}_{h} & & \\
& & 0_{m \times m} & \\
& & & \mathcal{I}_{h}
\end{array}\right],
$$

where the identity matrices $\mathcal{I}_{h}$ refer to the entries of the state vector that are related to the periodic modes. Consequently, the estimation of the output data contribution due to the periodic modes writes as

$$
\hat{y}_{k}^{\text {per }}=C^{V} S \hat{x}_{k}^{V}
$$

which is the desired estimate of the periodic subsignal.

In practice only the estimates of $C^{V}, A^{V}$ and $K_{k}^{V}$ are available, which are computed on data of finite length, e.g., after [14]. Consequently, an approximate Kalman state is used in (23) to estimate the periodic subsignal.

\subsection{Removal of the periodic subsignal by orthogonal projection}

The estimate of the periodic subsignal can be decoupled from the row space of output data by using an adequate projection. Different projection methods exist and are often used in the context of subspace-based system identification, e.g., see $[14,20]$. In the following, an orthogonal projection of the raw output data onto the orthogonal complement of the estimated periodic subsignal is carried out. It is proved that the resulting signal indeed represents only the structural system, and the periodic part of the combined state-space system is canceled out.

In these projections, Hankel matrices of the respective signals are used, which are defined as follows.

Definition 1. Let the samples $k=i, \ldots, j+N-1$ of a discrete signal $a_{k} \in \mathbb{R}^{b \times 1}$ be given with $i \leq j$. Then the corresponding block Hankel matrix $\mathcal{A}_{i \mid j}$ is defined as

$$
\mathcal{A}_{i \mid j}=\left[\begin{array}{cccc}
a_{i} & a_{i+1} & \ldots & a_{i+N-1} \\
a_{i+1} & a_{i+2} & \ldots & a_{i+N} \\
\vdots & \vdots & \ddots & \vdots \\
a_{j} & a_{j+1} & \ldots & a_{j+N-1}
\end{array}\right] \in \mathbb{R}^{(j-i+1) b \times N} .
$$

Analogously to subspace methods, Hankel matrices are built from the signals with a 'past' and 'future' time horizon. Let $p$ be a parameter that defines the time lags for the past and future horizons. Based on the raw output data $y_{k}$ (e.g., see (15) or (20)), the past and future data Hankel matrices are defined as

$$
\mathcal{Y}_{\text {raw }}^{-}=\frac{1}{\sqrt{N}} \mathcal{Y}_{0 \mid p-1}, \quad \mathcal{Y}_{\text {raw }}^{+}=\frac{1}{\sqrt{N}} \mathcal{Y}_{p \mid 2 p-1} \text {. }
$$

Similarly, the past and future data Hankel matrices of the estimated periodic subsignal $\hat{y}_{k}^{\text {per }}$ (see (23)) are defined as

$$
\boldsymbol{Y}_{\mathrm{per}}^{-}=\frac{1}{\sqrt{N}} \hat{\mathfrak{Y}}_{0 \mid p-1}^{\mathrm{per}}, \quad \boldsymbol{Y}_{\mathrm{per}}^{+}=\frac{1}{\sqrt{N}} \hat{\mathfrak{y}}_{p \mid 2 p-1}^{\mathrm{per}} .
$$

Furthermore, denote the past and future block-row matrix of Kalman filter states in the modal basis $\hat{x}_{k}^{V}($ see $(21))$ as

$$
\mathcal{X}^{-}=\frac{1}{\sqrt{N}} \hat{X}_{0 \mid 0}^{V}, \quad \mathcal{X}^{+}=\frac{1}{\sqrt{N}} \hat{\mathcal{X}}_{p \mid p}^{V}
$$

The rows of $\mathcal{X}^{-}, \mathcal{X}^{+} \in \mathbb{R}^{2(m+h) \times N}$ corresponding to the system part are denoted as $\mathcal{X}_{\text {sys }}^{-}, \mathcal{X}_{\text {sys }}^{+} \in \mathbb{R}^{2 m \times N}$, and corresponding to the periodic part as $\mathcal{X}_{\mathrm{per}}^{-}, \mathcal{X}_{\mathrm{per}}^{+} \in \mathbb{R}^{2 h \times N}$, respectively. The past and future Hankel matrices based on the innovations $e_{k}$ (see (19)-(20)) are defined as

$$
\mathcal{E}^{-}=\frac{1}{\sqrt{N}} \mathcal{E}_{0 \mid p-1}, \quad \mathcal{E}^{+}=\frac{1}{\sqrt{N}} \mathcal{E}_{p \mid 2 p-1} .
$$


Finally, the extended observability matrix of the combined system writes in the modal basis as

$$
\Gamma=\left[\begin{array}{c}
C^{V} \\
C^{V} A^{V} \\
\vdots \\
C^{V}\left(A^{V}\right)^{p-1}
\end{array}\right],
$$

and let $\Gamma_{\text {sys }}$ and $\Gamma_{\text {per }}$ be its columns corresponding to the system and to the periodic part, respectively. With this notation, the data Hankel matrices of the raw data and of the estimated periodic subsignal can be expressed in terms of the states and the innovations by recursion of the innovation state-space model (19)-(20) as follows.

Corollary 2 (Matrix output-only innovation state-space equations). It holds

$$
\begin{array}{lll}
\mathcal{Y}_{\text {raw }}^{-}=\Gamma \mathcal{X}^{-}+\mathcal{K} \mathcal{E}^{-}+\mathcal{E}_{\mathcal{K}}^{-}, & \mathcal{Y}_{\text {raw }}^{+}=\Gamma \mathcal{X}^{+}+\mathcal{K} \mathcal{E}^{+}+\mathcal{E}_{\mathcal{K}}^{+}, \\
\mathcal{Y}_{\text {per }}^{-}=\Gamma_{\text {per }} \mathcal{X}_{\text {per }}^{-}, & y_{\text {per }}^{+}=\Gamma_{\text {per }} \mathcal{X}_{\text {per }}^{+},
\end{array}
$$

where $\mathcal{K} \mathcal{E}^{-}$and $\mathcal{K} \mathcal{E}^{+}$are related to the innovation terms with $\mathcal{K} \in \mathbb{R}^{p r \times p r}$ being defined based on the steady state Kalman gain $K$, and $\mathcal{E}_{\mathcal{K}}^{-}$and $\mathcal{E}_{\mathcal{K}}^{+}$are remainder terms that are related to the difference to the actual non-steady state Kalman gain $K_{k}$, with

$$
\mathcal{K}=\left[\begin{array}{ccccc}
\mathcal{I}_{r} & 0 & 0 & \ldots & 0 \\
C K & \mathcal{I}_{r} & 0 & \ldots & 0 \\
C A K & C K & \mathcal{I}_{r} & \ldots & 0 \\
\ldots & \ldots & \ldots & \ldots & \ldots \\
C A^{p-2} K & C A^{p-3} K & C A^{p-4} K & \ldots & \mathcal{I}_{r}
\end{array}\right], \mathcal{K}_{l}=\left[\begin{array}{ccccc}
\mathcal{I}_{r} & 0 & 0 & \ldots & 0 \\
C K_{l} & \mathcal{I}_{r} & 0 & \ldots & 0 \\
C A K_{l} & C K_{l+1} & \mathcal{I}_{r} & \ldots & 0 \\
\ldots & \ldots & \ldots & \ldots & \ldots \\
C A^{p-2} K_{l} & C A^{p-2} K_{l+1} & C A^{p-3} K_{l+2} & \ldots & \mathcal{I}_{r}
\end{array}\right],
$$

where the l-th columns of $\mathcal{E}_{\mathcal{K}}^{-}$and $\mathcal{E}_{\mathcal{K}}^{+}$are, respectively,

$$
\left[\mathcal{E}_{\mathcal{K}}^{-}\right]_{l}=\left(\mathcal{K}_{l-1}-\mathcal{K}\right)\left[\mathcal{E}^{-}\right]_{l},\left[\mathcal{E}_{\mathcal{K}}^{+}\right]_{l}=\left(\mathcal{K}_{l-1+p}-\mathcal{K}\right)\left[\mathcal{E}^{+}\right]_{l}
$$

These remainder terms converge to zero as l grows since the non-steady state Kalman gain $K_{l}$ converges to the steady state gain $K$ during the transient phase, which is necessary due to possible errors in the initial estimates $\hat{x}_{0}$ and $P_{0}$. Note that the transient aspect of the non-periodic part of these remainder terms could be neglected since the respective part of the gain converges to its steady state limit fast, namely at exponential rate. However, since the periodic part converges only at a linear rate [33], they cannot be neglected without further analysis.

Equations (26) and (27) are required for the analysis of projections of the data Hankel matrices. In particular, the terms $\Gamma \mathcal{X}^{-}$and $\Gamma \mathcal{X}^{+}$in the raw data in (26) contain both system and periodic parts, yielding $\Gamma \mathcal{X}^{-}=\Gamma_{\mathrm{sys}} \mathcal{X}_{\mathrm{sys}}^{-}+\Gamma_{\mathrm{per}} \mathcal{X}_{\mathrm{per}}^{-}$ and $\Gamma \mathcal{X}^{+}=\Gamma_{\mathrm{sys}} \mathcal{X}_{\mathrm{sys}}^{+}+\Gamma_{\mathrm{per}} \mathcal{X}_{\mathrm{per}}^{+}$. In the proposed method the raw data matrix is projected on the orthogonal complement of the data matrix of the estimated periodic subsignal in order to remove the periodic part from the raw data. To this end, the projection matrices

$$
\begin{aligned}
& \mathcal{Y}_{\text {pro }}^{-}=\mathcal{Y}_{\text {raw }}^{-} / \mathcal{Y}_{\text {per }}^{-}{ }^{\perp}=\mathcal{Y}_{\text {raw }}^{-}-\mathcal{Y}_{\text {raw }}^{-} \mathcal{Y}_{\text {per }}^{-T^{T}}\left(\mathcal{Y}_{\text {per }}^{-} \mathcal{Y}_{\text {per }}^{-T}\right)^{\dagger} \mathcal{Y}_{\text {per }}^{-}, \\
& \mathcal{Y}_{\text {pro }}^{+}=\mathcal{Y}_{\text {raw }}^{+} / \mathcal{Y}_{\text {per }}^{+\perp}=\mathcal{Y}_{\text {raw }}^{+}-\mathcal{Y}_{\text {raw }}^{+} \mathcal{Y}_{\text {per }}^{+T}\left(\mathcal{Y}_{\text {per }}^{+} \mathcal{Y}_{\text {per }}^{+T}\right)^{\dagger} \mathcal{Y}_{\text {per }}^{+}
\end{aligned}
$$

are defined. With these projections, the periodic parts of the terms $\Gamma \mathcal{X}^{-}$and $\Gamma \mathcal{X}^{+}$in the raw data in (26) are removed asymptotically, as shown in the following theorem.

Theorem 3. The orthogonal projection of the raw data matrix onto the orthogonal complement of the data matrix of the estimated periodic subsignal yields the decomposition

$$
\begin{aligned}
& \mathcal{Y}_{\text {pro }}^{-}=\Gamma_{\text {sys }} \mathcal{X}_{\text {sys }}^{-}+\mathcal{K} \mathcal{E}^{-}+\mathcal{E}_{\mathcal{K}}^{-}+o(1), \\
& \mathcal{Y}_{\text {pro }}^{+}=\Gamma_{\text {sys }} \mathcal{X}_{\text {sys }}^{+}+\mathcal{K} \mathcal{E}^{+}+\mathcal{E}_{\mathcal{K}}^{+}+o(1),
\end{aligned}
$$

where o(1) is a matrix whose norm converges almost surely to zero when $N \rightarrow \infty$. 


\section{Proof: See Appendix C.}

Hence, the orthogonal projections (29)-(30) provide a reconstructed output signal where the periodic system parts are (asymptotically) removed. The samples $\hat{y}_{k}^{\text {pro }}$ of this signal can be recovered from the block rows of $\boldsymbol{y}_{\text {pro }}^{-}$or $\mathcal{Y}_{\text {pro }}^{+}$. Based on these signals, the subspace-based system identification can be used to estimate only the structural system modes, as detailed in the following section.

\subsection{Output-only subspace identification of the system part from $\mathcal{Y}_{\text {pro }}$}

In SSI-UPC [14], a projection of the future output data matrix onto its past yields the factorization into the observability matrix of the system and a Kalman filter state sequence. From the observability matrix, the system matrices $A$ and $C$ are obtained, and subsequently the modal parameters.

In the following, the modal parameters of the structural system are obtained from a projection of the data matrices $\mathcal{Y}_{\text {pro }}^{+}$and $\mathcal{Y}_{\text {pro }}^{-}$analogously to the UPC method, namely

$$
\mathcal{H}=\mathcal{Y}_{\text {pro }}^{+} / \mathcal{Y}_{\text {pro }}^{-}
$$

To investigate the properties of this projection with respect to the identification of the structural system, define the matrices $\mathcal{Y}_{\text {sys }}^{+}=\Gamma_{\text {sys }} \mathcal{X}_{\text {sys }}^{+}+\mathcal{K} \mathcal{E}^{+}$and $\mathcal{Y}_{\text {sys }}^{-}=\Gamma_{\text {sys }} \mathcal{X}_{\text {sys }}^{-}+\mathcal{K} \mathcal{E}^{-}$. These matrices would contain the outputs of the structural system without the contribution of the periodic excitation, see Appendix A. Note that they are not actually computed on data, nor do they contain the contribution of the transient part of the non-steady Kalman filter since it decays with $N$. With these definitions, the proposed subspace procedure in (31) yields the same projection as $\mathcal{Y}_{\text {sys }}^{+}$onto $\mathcal{Y}_{\text {sys }}^{-}$, as shown in the following theorem.

Theorem 4. The projection of the future projected data matrix $\mathcal{Y}_{\text {pro }}^{+}$onto its past $\mathcal{Y}_{\text {pro }}^{-}$yields the factorization

$$
\mathcal{H}=\mathcal{Y}_{\text {pro }}^{+} / \mathcal{Y}_{\text {pro }}^{-}=\mathcal{Y}_{\text {sys }}^{+} / \mathcal{Y}_{\text {sys }}^{-}+o(1)=\Gamma_{\text {sys }} \mathcal{X}_{\text {sys }}^{+} / \mathcal{Y}_{\text {sys }}^{-}+o(1),
$$

from where an estimate of the observability matrix $\Gamma_{\text {sys }}$ of the system part can be obtained.

Proof: See Appendix D.

Estimates of the system parameters from matrix $\mathcal{H}$ are shown to be consistent in the following corollary.

Corollary 5. The subspace method using $\mathcal{H}$ in Theorem 4 is consistent for the estimation of the system matrices and subsequently of the modal parameters of the structural system, i.e., they converge to the true parameters of the structural system for $N \rightarrow \infty$.

\section{Proof: See Appendix E.}

The estimates of $A^{\text {sys }}$ and $C^{\text {sys }}$ and subsequently the estimates of modal parameters can be computed from $\mathcal{H}$ in a classical way after Appendix B and (7).

Remark 6. Projections are a common tool for system identification with subspace methods. In the proposed approach, the orthogonal projections (29)-(30) were used to reconstruct an output signal, where the periodic parts are removed (Theorem 3), and which can be used for consistent system identification (Theorem 4 and Corollary 5). A similar result can be achieved by subtracting the estimated periodic subsignal from the raw data, instead of the proposed orthogonal projection. Defining $\mathcal{Y}_{\text {diff }}^{-}=\mathcal{Y}_{\text {raw }}^{-}-\mathcal{Y}_{\text {per }}^{-}$and $\mathcal{Y}_{\text {diff }}^{+}=\mathcal{Y}_{\text {raw }}^{+}-\mathcal{Y}_{\text {per }}^{+}$yields a similar decomposition as in Theorem 3 thanks to properties (26)-(27), and analogous results as in Theorem 4 and Corollary 5 can be proven for consistent identification using $\mathcal{H}=\mathcal{Y}_{\text {diff }}^{+} / \mathcal{Y}_{\text {diff }}^{-}$. 


\subsection{Numerically efficient implementation}

The projections to obtain $\mathcal{Y}_{\text {pro }}^{-}, \mathcal{Y}_{\text {pro }}^{+}$and $\mathcal{H}$ in (29), (30) and (31) may be costly in computational efforts. An efficient numerical implementation that avoids the explicit computation of these projections is described in this section. The $\mathcal{L} Q$ decomposition of the stacked $\mathcal{Y}_{\text {per }}$ and $\mathcal{Y}_{\text {raw }}$ writes

$$
\left[\begin{array}{c}
\mathcal{Y}_{\text {per }}^{-} \\
\mathcal{Y}_{\text {per }}^{+} \\
\hdashline \mathcal{Y}_{\text {raw }}^{-} \\
\mathcal{Y}_{\text {raw }}^{+}
\end{array}\right]=\left[\begin{array}{cc:cc}
\mathcal{L}_{11} & 0 & 0 & 0 \\
\mathcal{L}_{21} & \mathcal{L}_{22} & 0 & 0 \\
\hdashline \mathcal{L}_{31}^{-} & \mathcal{L}_{32} & \mathcal{L}_{33}^{-} & 0 \\
\mathcal{L}_{41} & \mathcal{L}_{42} & \mathcal{L}_{43} & \mathcal{L}_{44}
\end{array}\right]\left[\begin{array}{c}
Q_{1}^{T} \\
Q_{2}^{T} \\
\hdashline \mathcal{Q}_{3}^{T} \\
\mathcal{Q}_{4}^{T}
\end{array}\right]=\left[\begin{array}{c:c}
\mathcal{L}_{12,12} & 0 \\
\hdashline \mathcal{L}_{34,12} & \mathcal{L}_{34,34}
\end{array}\right]\left[\begin{array}{c}
Q_{12}^{T} \\
\hdashline Q_{34}^{T}
\end{array}\right] .
$$

The combined projected data matrices $\mathcal{Y}_{\text {pro }}^{-}$and $\mathcal{Y}_{\text {pro }}^{+}$from (29)-(30) can also be (asymptotically) expressed as follows, and plugging in (32) yields

$$
\begin{aligned}
{\left[\begin{array}{l}
\mathcal{Y}_{\text {pro }}^{-} \\
\mathcal{y}_{\text {pro }}^{+}
\end{array}\right] } & =\left[\begin{array}{c}
\mathcal{Y}_{\text {raw }}^{-} \\
\mathcal{Y}_{\text {raw }}^{+}
\end{array}\right] /\left[\begin{array}{c}
\mathcal{y}_{\text {per }}^{-} \\
\mathcal{y}_{\text {per }}^{+}
\end{array}\right]^{\perp} \\
& =\left(\mathcal{L}_{34,12} Q_{12}^{T}+\mathcal{L}_{34,34} Q_{34}^{T}\right)\left(I-Q_{12} \mathcal{L}_{12,12}^{T}\left(\mathcal{L}_{12,12} Q_{12}^{T} Q_{12} \mathcal{L}_{12,12}^{T}\right)^{-1} \mathcal{L}_{12,12} Q_{12}^{T}\right) \\
& =\left(\mathcal{L}_{34,12} Q_{12}^{T}+\mathcal{L}_{34,34} Q_{34}^{T}\right)\left(I-Q_{12} Q_{12}^{T}\right) \\
& =\mathcal{L}_{34,34} Q_{34}^{T}=\left[\begin{array}{cc}
\mathcal{L}_{33} & 0 \\
\mathcal{L}_{43} & \mathcal{L}_{44}
\end{array}\right]\left[\begin{array}{l}
Q_{3}^{T} \\
Q_{4}^{T}
\end{array}\right] .
\end{aligned}
$$

The projection from (31) yields thus

$$
\mathcal{H}=\mathcal{Y}_{\text {pro }}^{+} / \mathcal{Y}_{\text {pro }}^{-}=\left(\mathcal{L}_{43} Q_{3}^{T}+\mathcal{L}_{44} Q_{4}^{T}\right) Q_{3} \mathcal{L}_{33}^{T}\left(\mathcal{L}_{33} \mathcal{L}_{33}^{T}\right)^{-1} \mathcal{L}_{33} Q_{3}^{T}=\mathcal{L}_{43} Q_{3}^{T} .
$$

Since the observability matrix $\Gamma_{\text {sys }}$ is estimated from the column space of $\mathcal{H}$ (see Appendix B) and since $Q_{3}$ is an orthogonal matrix, $\mathcal{L}_{43}$ can directly be used to estimate $\Gamma_{\text {sys }}$, without explicitly performing the projection in (31). The proposed scheme is summarized in Algorithm 1.

Remark 7. When the number of sensors is bigger than the number of periodic modes, i.e., $r>2 h$, the estimated periodic subsignal from (23) contains $r-2 h$ redundant responses and $\operatorname{rank}\left(\mathcal{Y}_{\text {per }}\right)=2 h(p+1)$. Consequently $\mathcal{L}_{12,12}$ becomes rank deficient, while full rank is needed to obtain (33). In such a case, the periodic states selected directly from the rows of $\hat{x}_{k}^{V}$ can substitute the estimation of the periodic subsignal from (23) by

$$
\hat{y}_{k}^{\mathrm{per}}=\hat{x}_{k}^{\text {Vper }} .
$$

This leads to a reduction of the dimensions of $\mathcal{Y}_{\text {per }}^{-}$and $\mathcal{Y}_{\text {per }}^{+}$without changing the projected matrices $\mathcal{Y}_{\text {pro }}^{-}$and $\mathcal{Y}_{\text {pro, }}^{+}$, and consequently (33) holds.

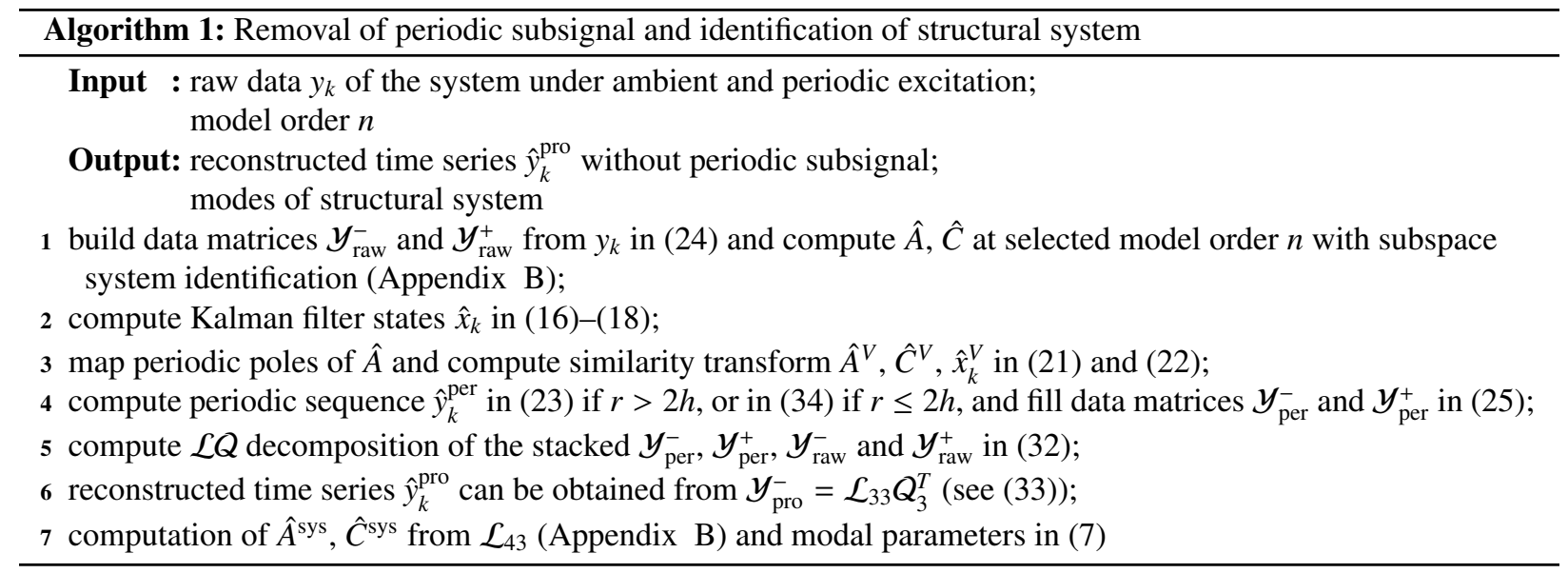




\section{Numerical validation}

In this section the proposed method is deployed first to remove the periodic mode information from the simulation of the chain system described in Section 2.3 and second to identify its structural modal parameters.

First, the adequacy of the combined state-space model (14)-(15), containing both system and periodic parts, is illustrated by comparing the exact system states to their computed Kalman filter counterparts. For this, output data of the mechanical system are simulated under both white noise and periodic excitation as well as output noise, using the discrete-time version of model (8)-(9). Then, the exact system and periodic states of model (14)-(15) are computed and transformed into the real-valued modal basis. To compare them to their estimates, the system matrices of the combined model are estimated at model order 14 from the simulated outputs, and the non-steady state Kalman filter states are computed with (16)-(18). To transform them into the modal basis of the exact states, an appropriate scaling of the estimated eigenvectors is needed. These scaling factors are obtained by relating the identified mode shapes to the theoretical ones.

In Figure 6, the exact states and computed Kalman states corresponding to the third structural mode of the chain system are shown, which is the mode closest to the periodic mode. In Figure 7, the exact states and computed Kalman states of the periodic mode are shown. It can be observed that the Kalman states of the structural mode are close to the exact states after about 5 samples, and after approximately 80 samples for the periodic mode. This suggests that a good approximation of the system states is obtained after a transient phase, where the non-steady state Kalman gain from (16) converges. Note that the non-steady state Kalman filter is able to estimate the states of the periodic mode accurately while the initial condition of those was not exact. This would not be possible using the converged steady state Kalman gain whose periodic part is zero.

Next, Algorithm 1 for the removal of the periodic part is applied. In Figure 8 the two highest PSD singular values
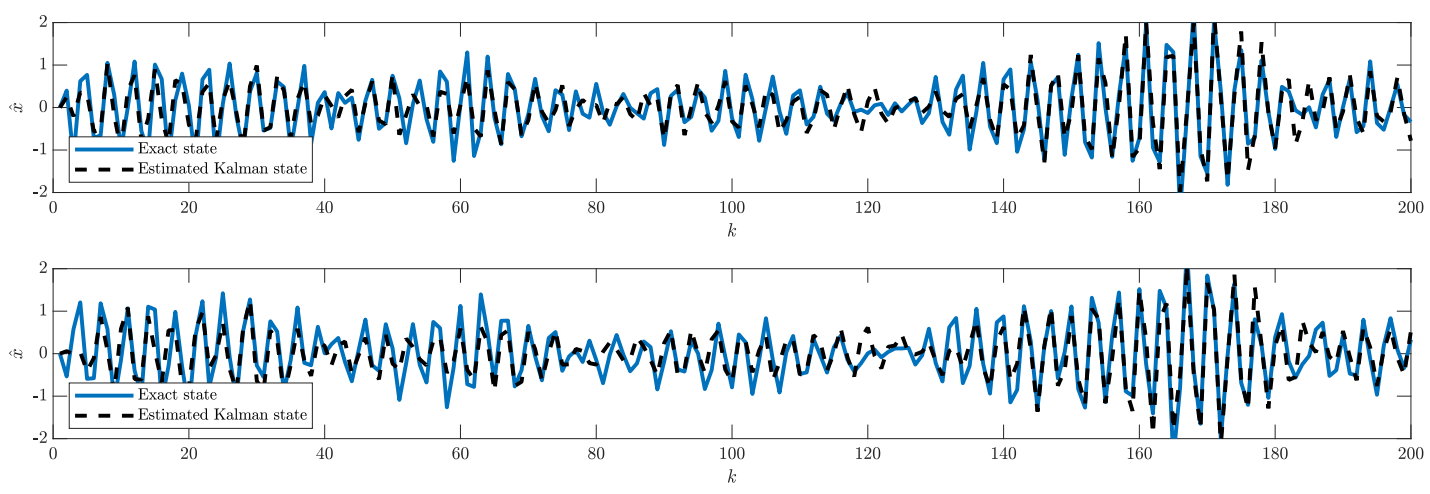

Figure 6: Exact states and computed Kalman states corresponding to the structural mode closest to the periodic mode.
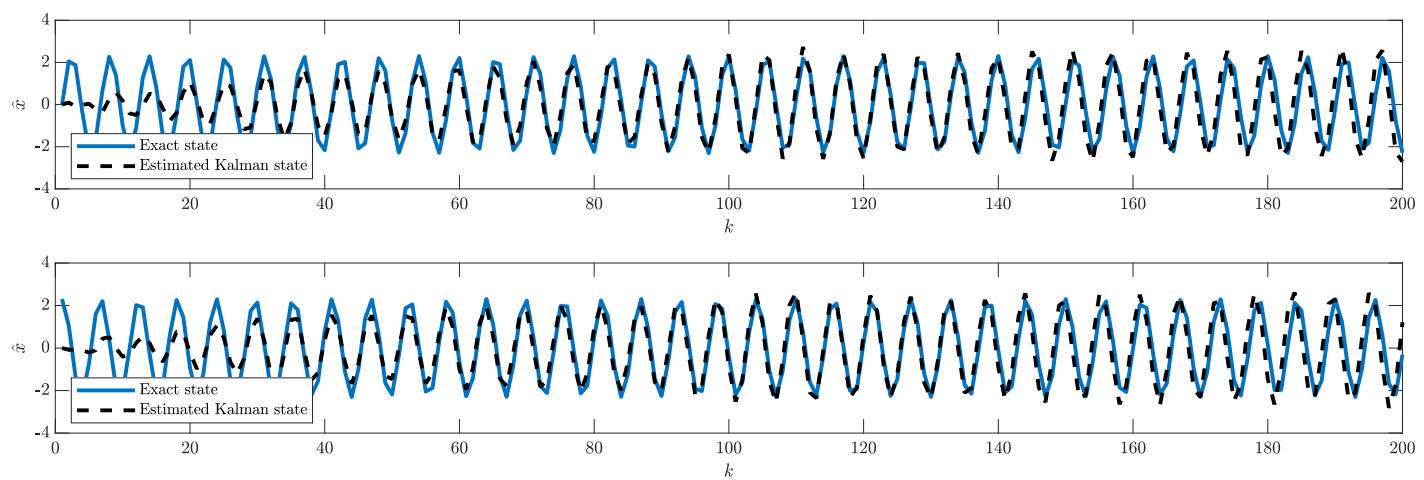

Figure 7: Exact states and computed Kalman states corresponding to the periodic mode. 

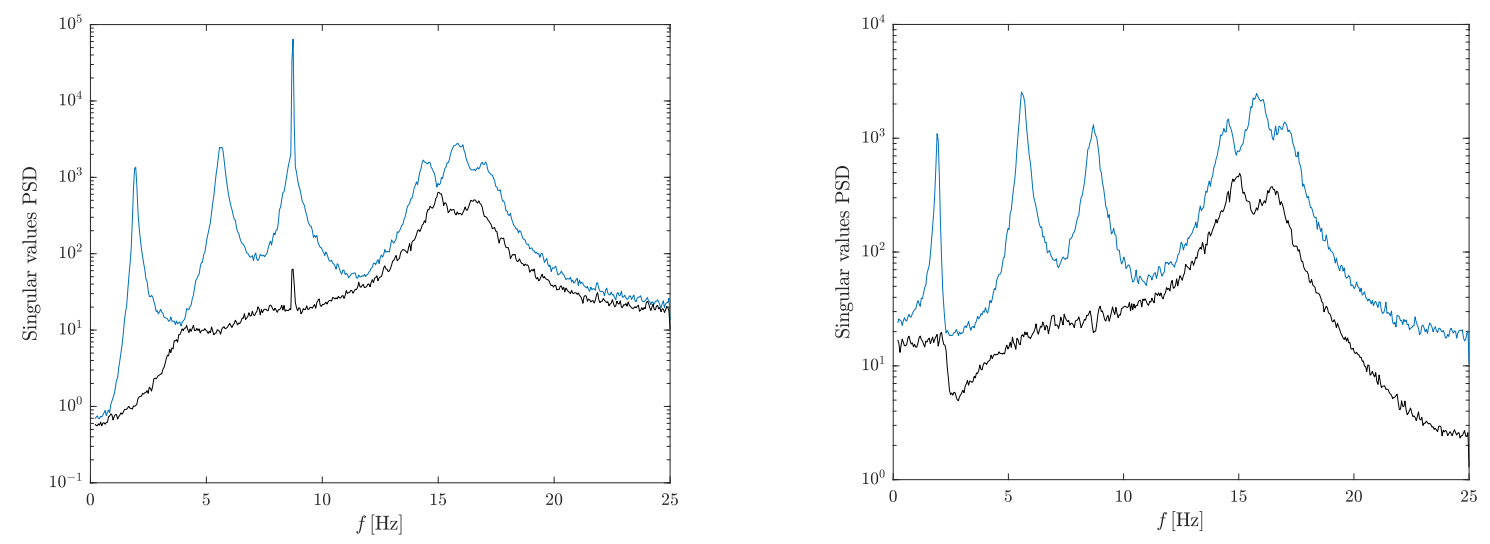

Figure 8: Two largest singular values of PSD matrix from raw data $y_{k}$ (left) and from projected data $\hat{y}_{k}^{\text {pro }}$ after removal of the periodic subsignal (right).
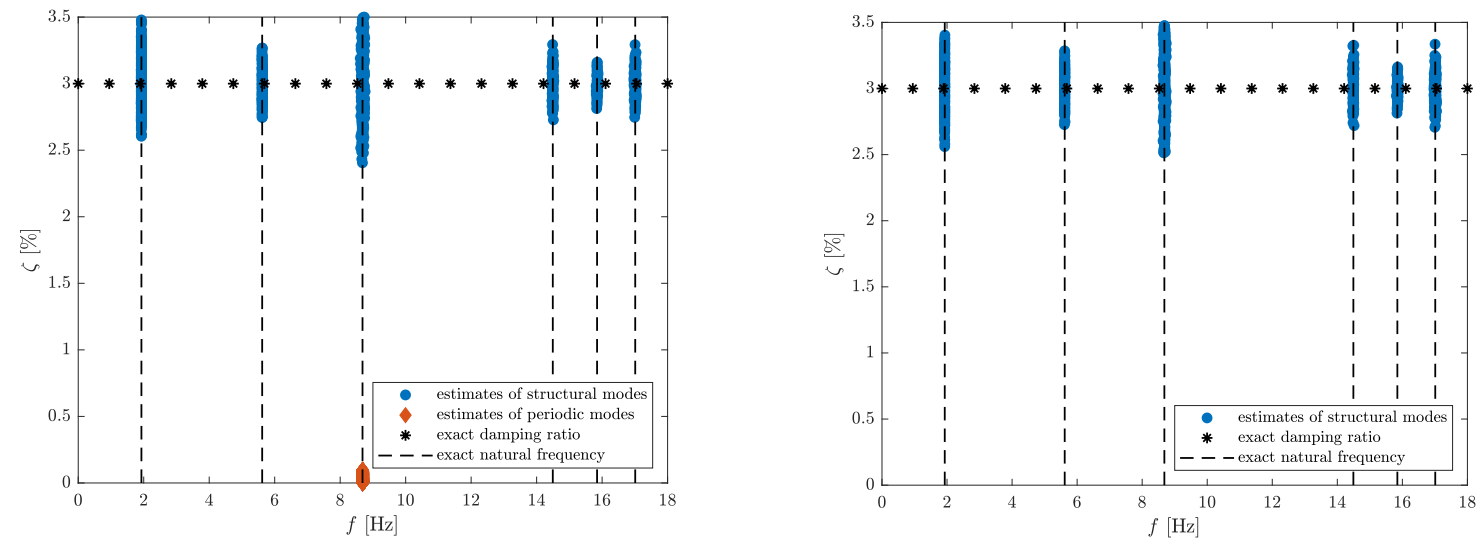

Figure 9: Estimates of natural frequencies and damping ratios from raw data $y_{k}$ (left) and from projected data $\hat{y}_{k}^{\text {pro }}$ after removal of the periodic subsignal (right) for the complete Monte Carlo simulation.

are shown that are computed from the raw data $y_{k}$ (left) and from the time series $\hat{y}_{k}^{\text {pro }}$ that is reconstructed from the projected data matrix $\mathcal{Y}_{\text {pro }}^{-}$after (29) (right). From Figure 8 it can be seen that the sharp peak corresponding to the periodic frequency at $8.69 \mathrm{~Hz}$ in Figure 8 (left) is cancelled in Figure 8 (right), and the resultant PSD plot resembles the reference case where no periodic inputs are present in Figure 2. Therefore it can be conjectured that the periodic information is removed from the raw data.

This is also verified in a Monte Carlo simulations, where the modal parameters are estimated first from the raw data and second from the projected data after removal of the periodic part with Algorithm 1. In Figure 9 the plots of the estimated frequencies versus damping ratios are shown for all the simulations. The periodic mode visible in Figure 9 (left) is identified with a low damping ratio and can easily be distinguished from modes of the structural system for the removal procedure. It can be observed that the periodic mode is indeed rejected in Figure 9 (right), and the estimates of natural frequencies and damping ratios are centered around the exact values from the model. Furthermore, it can be seen that the estimation uncertainties of the proposed method are similar as in the classical SSI-UPC, since the scattering of the estimated modal parameters is of the same magnitude before and after the rejection of the periodic subsignal. 


\section{Application}

In this section, two experimental cases are depicted to illustrate the performance of the proposed method. The first example is a plate subjected to a mix of random and periodic excitation in laboratory conditions. The second example is a full-scale test of a ship excited by random environmental load with interference from rotating machinery on-board.

\subsection{Plate with harmonics}

The experimental setup and the geometry of the plate are shown in Figure 10. Periodic excitation is applied by a shaker with a sinusoidal signal of $370 \mathrm{~Hz}$ continuously throughout the experiment. The measurements are sampled with $4096 \mathrm{~Hz}$ over a 120 seconds interval. The same experiment is also carried out without the periodic excitation in order to compare the modal parameter estimates with and without periodic excitation.

The frequency of the periodic signal is close to the first natural frequency of the plate, which is a particular challenge for system identification of experimental data [34]. In this context, the stochastic subspace identification of the raw data containing the responses to mixed random and periodic excitation is carried out with $p=20$ and model orders ranging from $n_{\min }=10$ to $n_{\max }=40$. In Figure 11 the resulting stabilization diagram of natural frequencies is shown. It can be seen that the periodic mode at $370 \mathrm{~Hz}$ and the close structural mode at $341 \mathrm{~Hz}$ cannot be identified below model order 20, since they are not separated. Other structural modes can already be identified at lower model orders, which suggests that the first structural mode may be perturbed by the periodic mode.

The frequency and damping ratio alignment of the periodic mode are presented in Figure 12 (left). It can be observed that its damping ratio is indeed small. The periodic mode estimate at model order 40 is then selected to estimate the periodic subsignal, used in the orthogonal projection in (29)-(30). In Figure 12 (right) the two largest PSD singular values are shown from the raw, the estimated periodic and the reconstructed system output data. It can
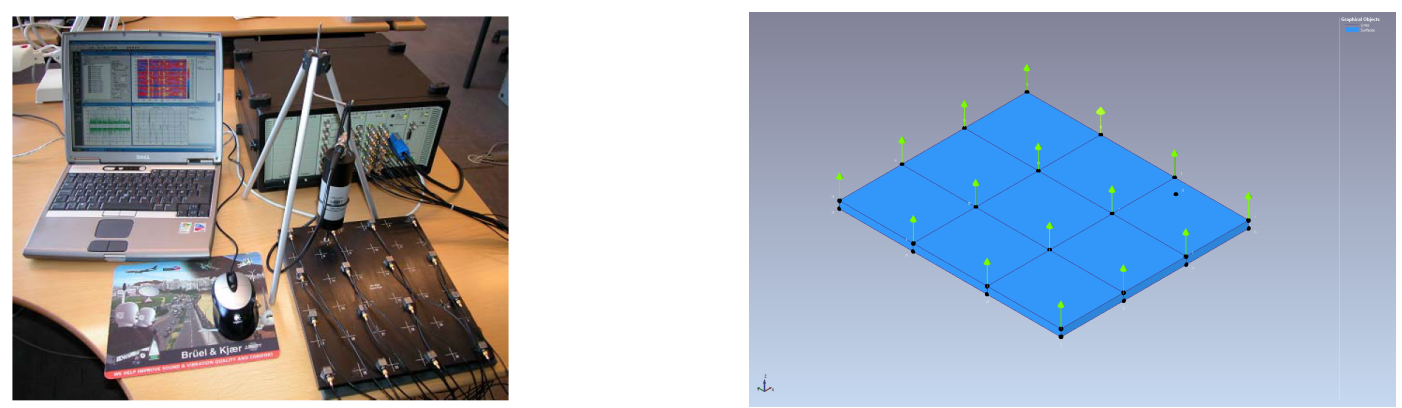

Figure 10: The experimental setup: plate with 16 acceleration channels, shaker, acquisition system (left). The plate model with 16 acceleration channels in ARTeMIS Modal Pro 6.0 (right).
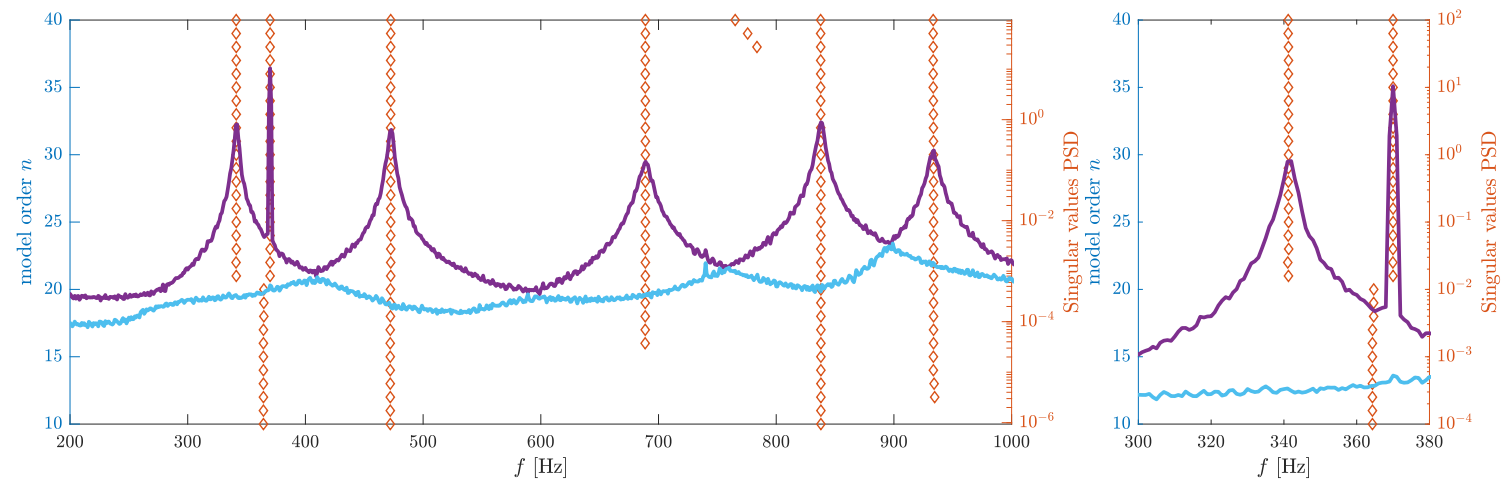

Figure 11: Stabilization diagram of the natural frequency estimates from the raw measurements of the plate containing both structural and periodic modes. 

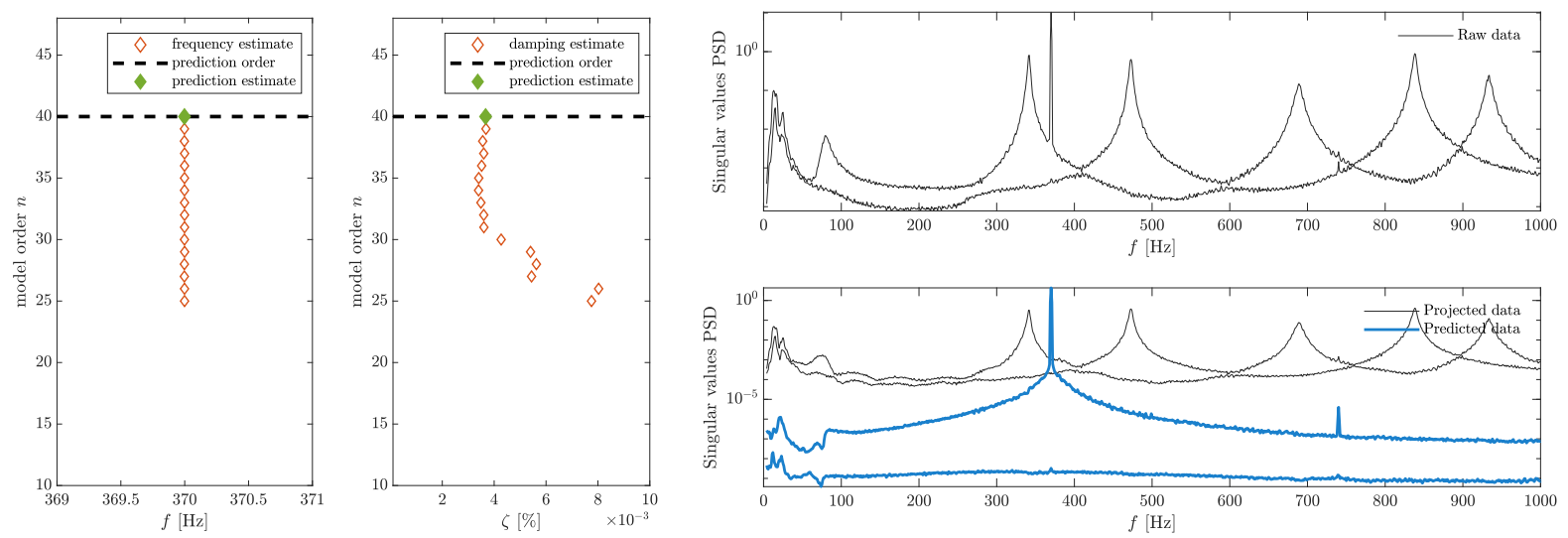

Figure 12: Left: Stable modal alignments of the natural frequency and damping ratio estimates of the periodic mode of the plate. Right: Two largest singular values of PSD from raw measurements (top), and estimated periodic signal and projected data (bottom).

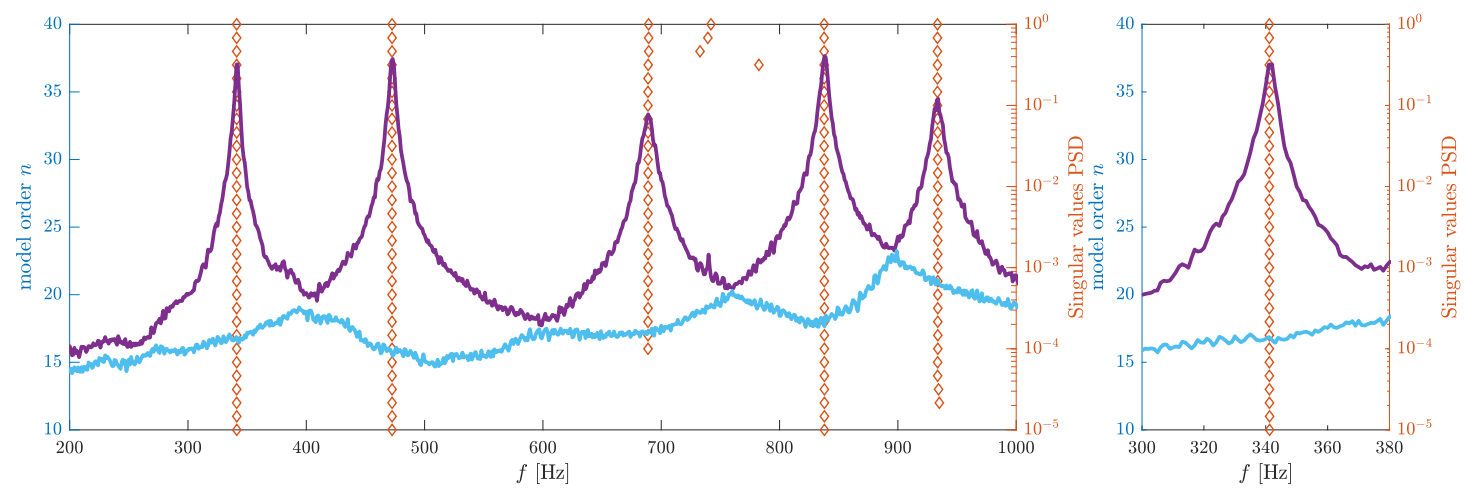

Figure 13: Stabilization diagram of the natural frequency estimates from measurements of the plate after the removal of the periodic subsignal.

be seen that the peaks of the PSD from the estimated periodic signal (blue line) match well with the periodic peaks of the PSD from the raw measurements.

Finally, the system identification results with the proposed method are presented after the removal of the periodic subsignal, corresponding to Algorithm 1. The corresponding stabilization diagram is shown in Figure 13. It can be observed that the periodic mode is no longer part of the estimated modes. In addition, the first natural frequency at $341 \mathrm{~Hz}$ is better estimated, namely already for much lower model orders compared to Figure 11, after the periodic part is removed.

A detailed comparison of the modal alignments for the natural frequencies and the damping ratios of the first mode estimated before and after the removal of the periodic subsignal is shown in Figure 14, where the results are also compared to the reference estimates from the plate experiment with only random and no periodic excitation. The estimated modal parameters are close to their counterparts estimated from the random response. While the estimated natural frequency and damping ratio are closer to their equivalent random response estimates after the removal of the periodic subsignal in Figure 14 (right) than before (left), the change in frequency towards its reference value is very small ( $0.05 \%$ of the value) and may not be significant. The change in damping ratio towards its reference value, however, is more significant (14\% of the reference value), indicating a less biased damping estimate of the first mode. For both the frequency and damping ratio, the alignments of the first mode after the removal of the periodic subsignal are more stable and start at a lower model order in Figure 14 (right) than before (left).

In Table 2 the alignment means of the first six modes are shown for the different experimental cases. With respect to the reference estimates from the random response, the frequency estimates before and after the removal of the periodic subsignal show very small differences that are of the same order for all modes. The differences in the 

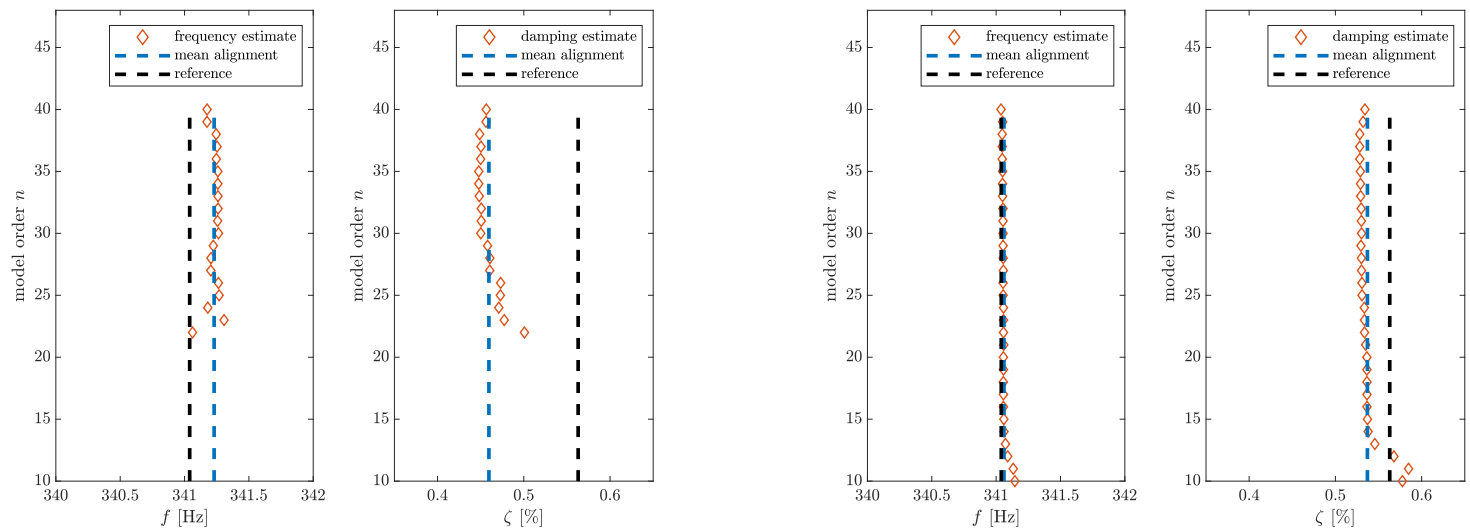

Figure 14: Modal alignments of the natural frequency and damping ratio estimates for the first structural mode from measurements before (left) and after (right) the removal of the periodic subsignal.

Table 2: Modal parameters of the plate without periodic excitation, and with periodic excitation estimated before and after removal of the periodic subsignal.

\begin{tabular}{llllllll}
\hline & Data type/Mode & & 1 & 2 & 3 & 4 & 5 \\
\hline \multirow{2}{*}{ random (reference) } & $f[\mathrm{~Hz}]$ & 341.04 & 472.12 & 688.34 & 837.03 & 933.62 & 1382.04 \\
& $\zeta[\%]$ & 0.563 & 0.494 & 0.757 & 0.453 & 0.569 & 0.971 \\
\hline \multirow{2}{*}{ mixed random and periodic } & $f[\mathrm{~Hz}]$ & 341.23 & 472.34 & 688.80 & 837.90 & 933.61 & 1381.62 \\
& $\zeta[\%]$ & 0.459 & 0.482 & 0.840 & 0.406 & 0.517 & 0.756 \\
\hline mixed random and periodic after removal of the & $f[\mathrm{~Hz}]$ & 341.06 & 472.49 & 688.91 & 837.79 & 932.81 & 1381.08 \\
periodic subsignal in (29) & $\zeta[\%]$ & 0.537 & 0.466 & 0.721 & 0.392 & 0.503 & 0.841 \\
\hline
\end{tabular}

damping estimates are naturally larger, and it can be seen that the estimates after the removal are either close to the values before the removal, or closer to the reference values.

From these results it can be concluded that the removal of the periodic subsignal leads to more stable alignments of the structural mode close to the periodic one, and the damping estimates of some of the modes (in particular of the mode close to the periodic one) are closer to the reference values.

\subsection{Ship in operation}

The considered structure is a roll-on roll-off ship on a test trail [35] that is subjected to random wind and wave loads interfered with periodic excitation from the propellers and the engine. Output accelerations are measured with 16 channels and are sampled with $128 \mathrm{~Hz}$ for 5400 seconds. The geometry of the ship with the measured degrees of freedom is illustrated in Figure 15. Prior to the analysis the data are decimated to $8 \mathrm{~Hz}$.
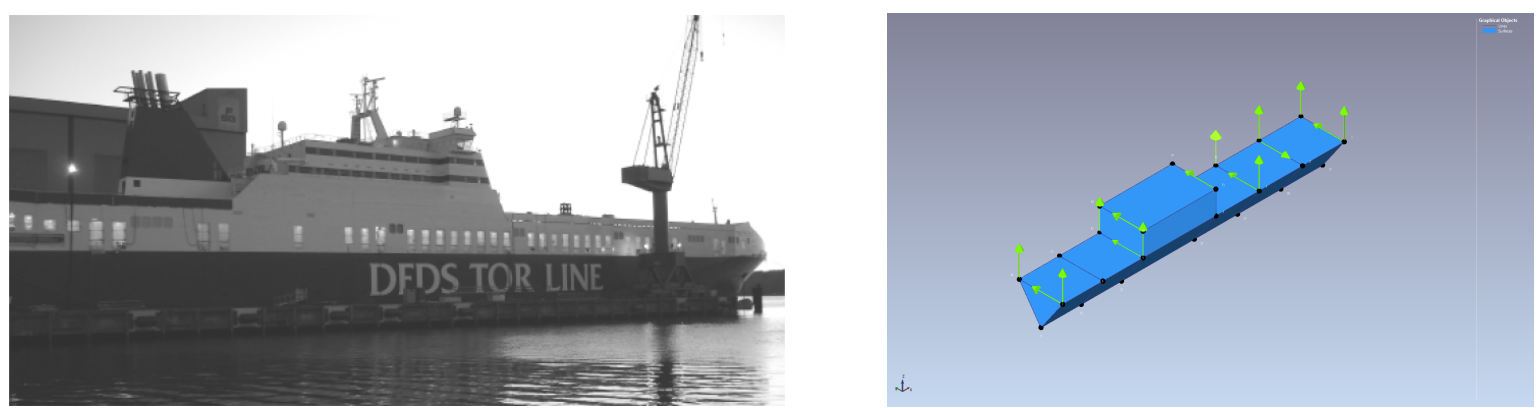

Figure 15: The ship at the Flensburg shipyard (left). The geometry of the ship with 16 acceleration channels in ARTeMIS Modal Pro 6.0 (right) 


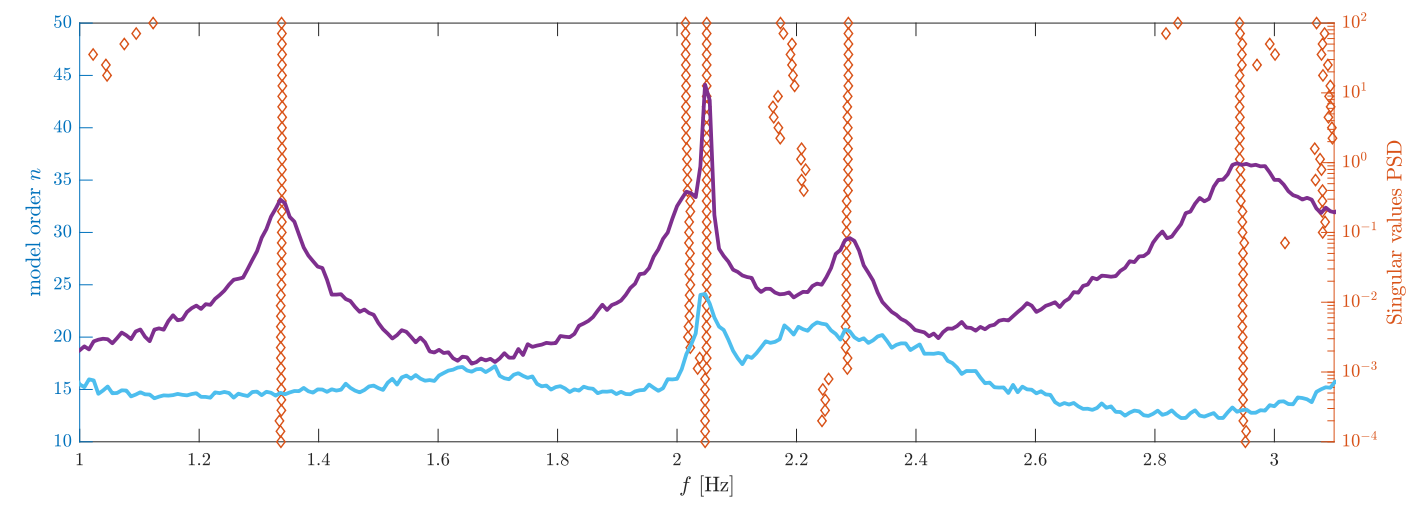

Figure 16: Stabilization diagram of the natural frequency estimates from the raw measurements of the ship containing both structural and periodic modes.
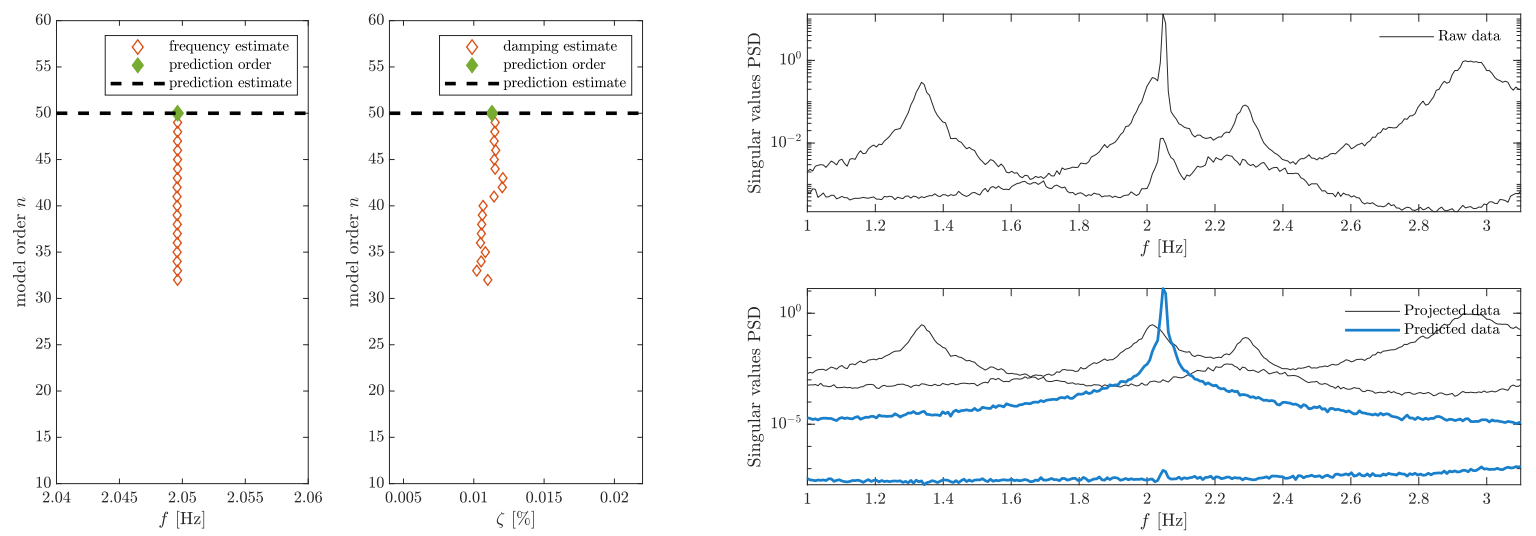

Figure 17: Left: Stable modal alignments of the natural frequency and damping ratio estimates of the periodic mode at $2.05 \mathrm{~Hz}$. Right: Two largest singular values of PSD from raw measurements (top), and estimated periodic signal and projected data (bottom).

Similar to the plate example from the previous section, the modal parameters are estimated from the data subjected to the mixed random/periodic excitation first. For this purpose the UPC algorithm is used with $p=25$ and model orders ranging from $n_{\min }=10$ to $n_{\max }=50$. The resulting stabilization diagram of natural frequencies is shown in Figure 16. It can be observed that the periodic frequency at $2.05 \mathrm{~Hz}$ is close to a structural mode of the ship whose estimation is possibly perturbed by the periodic mode.

A zoom on the modal alignment of the periodic mode is presented in Figure 17 (left). It can be observed that the damping ratio estimates of the periodic mode are low, which distinguishes it from the structural modes. Subsequently, the periodic mode at the model order 50 is selected for the removal of the periodic subsignal with (29). In Figure 17 (right) the two largest PSD singular values are shown of the raw, the estimated periodic and the reconstructed structural system output data. It can be observed that the peak of the PSD from the estimated periodic subsignal coincides well with the peak of the periodic mode from the raw measurements. Moreover the reconstructed output data contain no high energy frequency content at $2.05 \mathrm{~Hz}$, suggesting that the periodic mode information is removed.

Subsequently, results from the system identification with the proposed method are presented in Figure 18 after the removal of the periodic subsignal. They clearly illustrate that the periodic mode at $2.05 \mathrm{~Hz}$ is successfully removed from the data. A detailed comparison of the modal alignments of the close structural mode estimated from the measurements before and after the removal of the periodic subsignal is shown in Figure 19. Deviation of both natural frequencies and damping ratio estimates from the mean values of their modal alignments is lower when the periodic information is removed, suggesting that the proposed approach is beneficial in a practical modal analysis application. Moreover, the structural mode is already identified at lower model orders after the removal. 


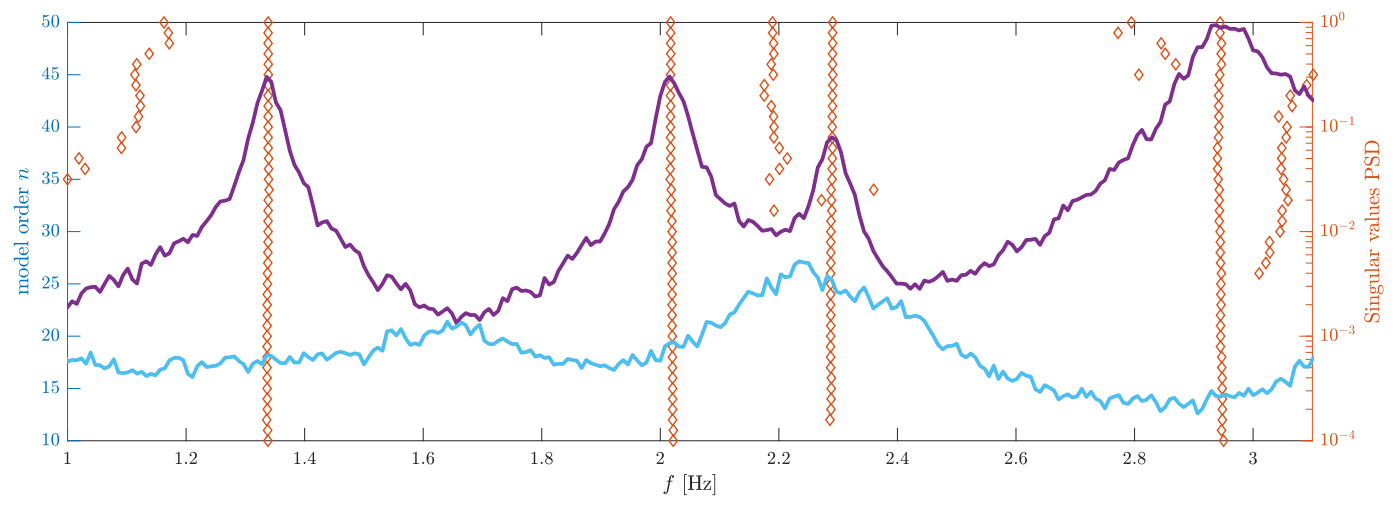

Figure 18: Stabilization diagram of the natural frequency estimates from measurements of the ship after the removal of the periodic subsignal.
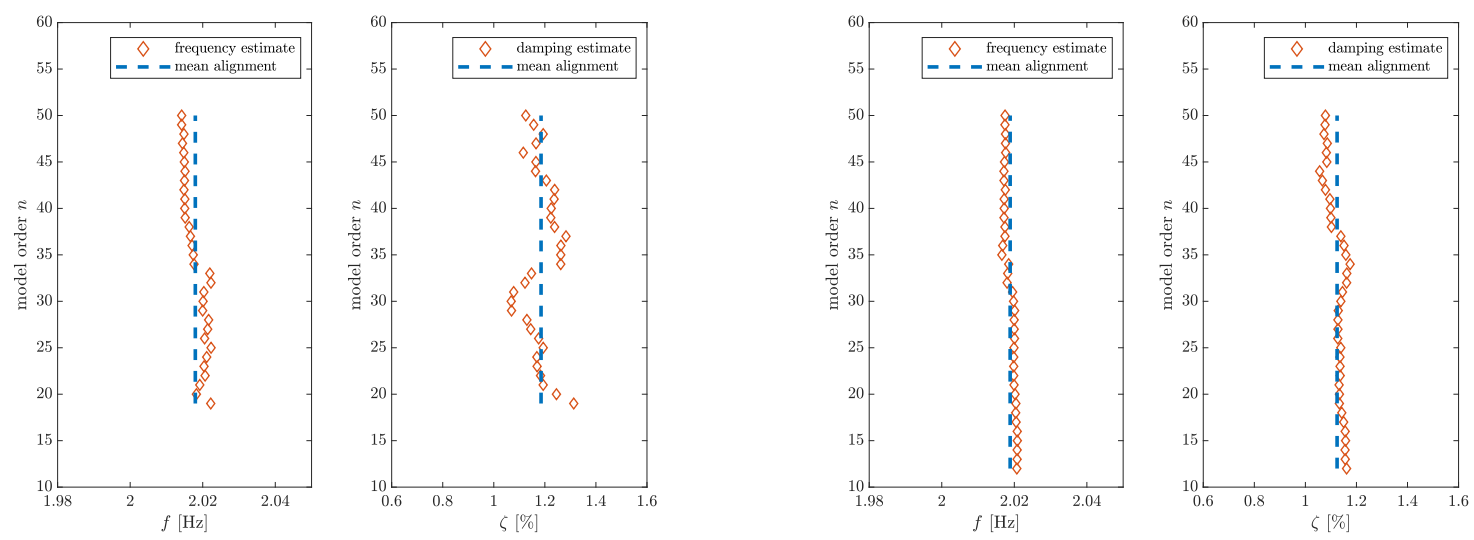

Figure 19: Modal alignments of the natural frequency and damping ratio estimates for the second structural mode (close to the periodic mode) from measurements before (left) and after (right) the removal of the periodic subsignal.

\section{Conclusion}

In this paper, a subspace framework has been derived for the estimation of the structural modes of a mechanical system under both ambient and periodic excitation. This approach consists of three steps, starting with a classical output-only SSI that provides initial estimates of the system matrices. These matrices are used in the second step to compute a sequence of non-steady Kalman states in the modal basis in order to estimate the periodic subsignal from the periodic modes. In the final step, the raw output data is projected onto the orthogonal complement of the estimated periodic signal, yielding a new subspace method for the identification of the structural modes while rejecting the periodic modes from the data.

Besides the development of the algorithmic procedure, a few theoretical results have been proved. First, it has been shown that the considered mechanical model under both ambient and periodic excitation is equivalent up to a similarity transform to the stationary modeling proposed in [23], where the stochastic and periodic subsystems are decoupled. This validates the first step of the developed method and yields stability properties of the Kalman state estimates. Second, the rejection of the periodic information from the raw signal has been explicitly formulated. Using the resulting signal for a UPC-like projection of its future time horizon onto its past, the factorization into the observability matrix and Kalman states of the structural system - without any periodic parts - has been proved to hold asymptotically. Consequently, consistency of the proposed SSI method has been shown.

Both the modeling and the removal of the periodic subsignal have been validated on simulations of a chain system, and the consistency of the identification was illustrated on Monte Carlo simulations. Furthermore, the proposed method was demonstrated on experimental data under both ambient and periodic excitation, namely on an aluminum 
plate excited by a shaker in the lab and on a ship in operation excited by the combination of environmental load and the interference from the rotation of engine and propellers. In both cases the periodic frequency was close to a natural frequency of the structure. The results illustrate that the periodic subsignal has been removed successfully, and that it led to more consistent modal parameter estimates of the structural modes with modal alignments that stabilize from a lower model order. Future work includes the uncertainty analysis of the algorithm.

\section{Acknowledgments}

Qinghua Zhang is acknowledged for discussions about the stability properties of the Kalman filter. Niels-Jørgen Jacobsen from Brüel \& Kjær is acknowledged for carrying out the experimental tests on the plate and sharing its results. Sven-Erik Rosenow, Santiago Uhlenbrock and Günther Schlottmann from University of Rostock are acknowledged for sharing the measurements of the ship.

\section{Appendix A. Theoretical innovation model and stability of Kalman filter}

In the following it is shown that the considered combined state-space model (14)-(15) corresponds to the theoretical innovation model

$$
\begin{aligned}
{\left[\begin{array}{c}
z_{k+1}^{s} \\
z_{k+1}^{d}
\end{array}\right] } & =\left[\begin{array}{cc}
A_{s} & 0 \\
0 & A_{d}
\end{array}\right]\left[\begin{array}{c}
z_{k}^{s} \\
z_{k}^{d}
\end{array}\right]+\left[\begin{array}{c}
K \\
0
\end{array}\right] e_{k}, \\
y_{k} & =\left[\begin{array}{ll}
C_{s} & C_{d}
\end{array}\right]\left[\begin{array}{c}
z_{k}^{s} \\
z_{k}^{d}
\end{array}\right]+e_{k} .
\end{aligned}
$$

This model was analyzed in [23], where observability of the pair $\left(C_{d}, A_{d}\right)$ is required, as well as observability and controllability of the stochastic subsystem. The theoretical innovation $e_{k}$ has finite fourth order moments. Under these conditions, stability of the Kalman filter is given, and finite fourth order moments of the states can be assumed.

First it is shown that there exists an invertible matrix $T$, such that

$$
T^{-1}\left[\begin{array}{cc}
A^{\text {sys }} & A^{\mathbf{b}} \\
0 & A^{\text {per }}
\end{array}\right] T=\left[\begin{array}{cc}
A^{\text {sys }} & 0 \\
0 & A^{\text {per }}
\end{array}\right], \quad \text { with } T=\left[\begin{array}{cc}
I & T_{12} \\
0 & I
\end{array}\right], T^{-1}=\left[\begin{array}{cc}
I & -T_{12} \\
0 & I
\end{array}\right] .
$$

Matrix $T_{12} \in \mathbb{R}^{2 m \times 2 h}$ (and thus matrix $T$ ) is constructed by multiplying out the left expression, leading to the necessary condition $A^{\text {sys }} T_{12}-T_{12} A^{\text {per }}+A^{\mathbf{b}}=0$. This Sylvester equation has a unique solution since the eigenvalues of $A^{\text {sys }}$ (inside the unitary circle) are distinct from the eigenvalues of $A^{\text {per }}$ (on the unitary circle). The solution is given through $\left[\left(I_{2 h} \otimes A^{\text {sys }}\right)-\left(A^{\text {perT }} \otimes I_{2 m}\right)\right] \operatorname{vec}\left(T_{12}\right)=-\operatorname{vec}\left(A^{\mathbf{b}}\right)$, where $\otimes$ denotes the Kronecker product and vec $(\cdot)$ the column stacking vectorization operator. Pre-multiplying (14) by $T^{-1}$ leads to the transformed state-space model

$$
\begin{aligned}
& {\left[\begin{array}{c}
\tilde{x}_{k+1}^{\text {sys }} \\
\tilde{x}_{k+1}^{\mathrm{per}}
\end{array}\right]=\left[\begin{array}{cc}
A^{\text {sys }} & 0 \\
0 & A^{\text {per }}
\end{array}\right]\left[\begin{array}{c}
\tilde{x}_{k}^{\text {sys }} \\
\tilde{x}_{k}^{\text {per }}
\end{array}\right]+\left[\begin{array}{c}
w_{k} \\
0
\end{array}\right],} \\
& y_{k}=\left[\begin{array}{ll}
C^{\text {sys }} & \left(C^{\text {sys }} T_{12}+C^{\text {per }}\right)
\end{array}\right]\left[\begin{array}{l}
\tilde{x}_{k}^{\text {sys }} \\
\tilde{x}_{k}^{\text {per }}
\end{array}\right]+v_{k},
\end{aligned}
$$

with the transformed states

$$
\left[\begin{array}{c}
\tilde{x}_{k}^{\text {sys }} \\
\tilde{x}_{k}^{\text {per }}
\end{array}\right]=T^{-1}\left[\begin{array}{c}
x_{k}^{\mathrm{sys}} \\
x_{k}^{\mathrm{per}}
\end{array}\right]=\left[\begin{array}{c}
x_{k}^{\mathrm{sys}}-T_{12} x_{k}^{\mathrm{per}} \\
x_{k}^{\mathrm{per}}
\end{array}\right] .
$$

Note that the process noise term in (A.3) remains the same after the similarity transform. Finally, assuming that the stochastic subsystem is observable and controllable by the noise, and assuming that the periodic subsystem is observable, the theoretical innovation model corresponding to (A.3)-(A.4) is indeed given by (A.1)-(A.2). Thus, following [23], the Kalman filter applied to data from the considered combined state-space model (14)-(15) is stable, and the fourth moments of the Kalman filter states and innovations are bounded [31]. Due to the stability of the Kalman filter, the same holds also when applying the non-steady state Kalman gain.

Note that regarding the states, (A.3) shows that there is a state space basis in which the periodic states $x_{k}^{\text {per }}$ (or equivalently $\tilde{x}_{k}^{\text {per }}$ ) are decoupled from the structural states $\tilde{x}_{k}^{\text {sys }}$, unlike the physical structural states $x_{k}^{\text {sys }}$ of the mechanical model in (14)-(15). Due to the block diagonal structure of the state transition matrix in (A.3), this is also the case for the modal basis. 


\section{Appendix B. Subspace system identification}

Assume the matrix $\hat{\mathcal{H}}$ enjoys (asymptotically) the factorization property into $\mathcal{H}=\Gamma X$ where $\Gamma \in \mathbb{R}^{(p+1) r \times n}$ is defined as

$$
\Gamma=\left[\begin{array}{c}
C \\
C A \\
\vdots \\
C A^{p}
\end{array}\right],
$$

and $X$ are the system states which are dependent on the chosen projection algorithm [14]. In practice, the estimate of the observability matrix $\Gamma$ is computed from the SVD of $\mathcal{H}$ estimated from the measured data sequences

$$
\hat{\mathcal{H}}=\left[\begin{array}{ll}
U_{1} & U_{2}
\end{array}\right]\left[\begin{array}{cc}
D_{1} & 0 \\
0 & D_{2}
\end{array}\right]\left[\begin{array}{l}
V_{1}^{T} \\
V_{2}^{T}
\end{array}\right],
$$

where an estimate of $\Gamma$ is taken as $\hat{\Gamma}=U_{1} D_{1}^{1 / 2}$. Matrices $U_{1}$ and $V_{1}$ are the left and right singular vectors corresponding to first $n$ non-zero singular values $D_{1}$ and $U_{2}$ with $V_{2}$ are the left and right kernel of $\hat{\mathcal{H}}$ where $D_{2} \rightarrow 0$. The estimates $\hat{A}$ and $\hat{C}$ can be computed in a least-square sense from the shift invariance property of $\hat{\Gamma}$.

\section{Appendix C. Proof of Theorem 3}

The first projection in Theorem 3 yields

$$
\begin{aligned}
\mathcal{Y}_{\text {pro }}^{-}=\mathcal{Y}_{\text {raw }}^{-} / \mathcal{Y}_{\text {per }}^{-}{ }^{\perp} & =\left(\Gamma_{\text {sys }} \mathcal{X}_{\text {sys }}^{-}+\Gamma_{\text {per }} \mathcal{X}_{\text {per }}^{-}+\mathcal{K} \mathcal{E}^{-}+\mathcal{E}_{\mathcal{K}}^{-}\right) / \mathcal{Y}_{\text {per }}^{-}{ }^{\perp} \\
& =\Gamma_{\text {sys }} \mathcal{X}_{\text {sys }}^{-} / \mathcal{Y}_{\text {per }}^{-}{ }^{\perp}+\underbrace{\Gamma_{\text {per }} \mathcal{X}_{\text {per }}^{-} / \Gamma_{\text {per }} \mathcal{X}_{\text {per }}^{-}}_{=0}+\mathcal{K} \mathcal{E}^{-} / \mathcal{Y}_{\text {per }}^{-}{ }^{\perp}+\mathcal{E}_{\mathcal{K}}^{-} / \mathcal{Y}_{\text {per }}^{-}{ }^{\perp},
\end{aligned}
$$

where the second term cancels out since an orthogonal projection yields $\mathcal{A} / \mathcal{A}^{\perp}=0$ for any matrix $\mathcal{A}$. The analysis of the last two terms requires a deeper insight into the properties of the estimated innovations and Kalman filter states. First, $\mathcal{E}^{-} \mathcal{X}_{\text {per }}^{-}{ }^{T} \rightarrow 0$ is shown to simplify the third term, and second, $\mathcal{E}_{\mathcal{K}}^{-} \mathcal{X}_{\text {per }}^{-} \rightarrow 0$ is shown for the fourth term. For this we suppose that $y_{k}$ and $\hat{x}_{k}$ have uniformly bounded fourth order moments, which is justified in Appendix A. Finally, the first term is simplified by showing $\mathcal{X}_{\text {sys }}^{-} \mathcal{X}_{\text {per }}^{-T} \rightarrow 0$.

\section{Proof of $\mathcal{E}^{-} \mathcal{X}_{\mathrm{per}}^{-T} \rightarrow 0$}

First a few mathematical notations: Consider the $\sigma$-algebra $\mathcal{F}_{k}$ generated by the observations $y_{1}, \ldots, y_{k}$. The collection of $\sigma$-algebra $\mathcal{F}_{k}$ is increasing, i.e., $\mathcal{F}_{k} \subset \mathcal{F}_{k+1}$. The innovation $e_{k}$ at time $k$ is $\mathcal{F}_{k}$ measurable, whereas $\hat{x}_{k}$ is $\mathcal{F}_{k-1}$ measurable. Since $\mathrm{E}\left(e_{k} \mid \mathcal{F}_{k-1}\right)=\mathrm{E}\left(e_{k} \mid Y_{1}, \ldots, Y_{k-1}\right)=0$, the innovations are uncorrelated with the past Kalman states (and also with the past outputs) [14], i.e.,

$$
\mathrm{E}\left(e_{k} \hat{x}_{k-j}^{T}\right)=0, \forall j \geq 0,
$$

which holds since $\mathrm{E}\left(e_{k} \hat{x}_{k-j}^{T}\right)=\mathrm{E}\left(\mathrm{E}\left(e_{k} \hat{x}_{k-j}^{T} \mid \mathcal{F}_{k-1}\right)\right)=\mathrm{E}\left(\mathrm{E}\left(e_{k} \mid \mathcal{F}_{k-1}\right) \hat{x}_{k-j}^{T}\right)=\mathrm{E}\left(0 . \hat{x}_{k-j}^{T}\right)=0$. Then, considering $\sup _{k} \mathrm{E}\left(\left\|e_{k}\right\|^{4}+\left\|\hat{x}_{k}\right\|^{4}\right)<C<\infty$, by Theorem 2.8 in [36],

$$
\mathcal{E}^{-} \mathcal{X}^{-T}=\frac{1}{N}\left[\begin{array}{cccc}
e_{0} & e_{1} & \vdots & e_{N-1} \\
e_{1} & e_{2} & \vdots & e_{N} \\
\vdots & \vdots & \vdots & \vdots \\
e_{p-1} & e_{p} & \vdots & e_{p+N-2}
\end{array}\right]\left[\begin{array}{c}
\hat{x}_{0}^{T} \\
\hat{x}_{1}^{T} \\
\vdots \\
\hat{x}_{N-1}^{T}
\end{array}\right]=\left[\begin{array}{c}
\frac{1}{N} \sum e_{l} \hat{x}_{l}^{T} \\
\frac{1}{N} \sum e_{l+1} \hat{x}_{l}^{T} \\
\vdots \\
\frac{1}{N} \sum e_{l+p-1} \hat{x}_{l}^{T}
\end{array}\right] \longrightarrow 0
$$


with probability 1 , in particular $\mathcal{E}^{-} \mathcal{X}_{\mathrm{per}}^{-T}=o(1)$. It follows $\mathcal{E}^{-} \mathcal{Y}_{\mathrm{per}}^{-T}=\mathcal{E}^{-} \mathcal{X}_{\mathrm{per}}^{-T} \Gamma_{\mathrm{per}}^{T}=o(1)$. Since $\left(\mathcal{Y}_{\mathrm{per}}^{-} \mathcal{Y}_{\mathrm{per}}^{-T}\right)^{\dagger}=$ $\left(\Gamma_{\mathrm{per}}^{T}\right)^{\dagger}\left(\mathcal{X}_{\mathrm{per}}^{-} \mathcal{X}_{\mathrm{per}}^{-T}\right)^{-1}\left(\Gamma_{\mathrm{per}}\right)^{\dagger}$ is bounded due to the distinct periodic eigenvalues and the resulting independence of the periodic state components, the third term in (C.1) yields

$$
\mathcal{K} \mathcal{E}^{-} / \mathcal{Y}_{\text {per }}^{-}{ }^{\perp}=\mathcal{K} \mathcal{E}^{-}-\mathcal{K} \mathcal{E}^{-} \mathcal{Y}_{\mathrm{per}}^{-{ }^{T}}\left(\boldsymbol{y}_{\mathrm{per}}^{-} \boldsymbol{Y}_{\mathrm{per}}^{-{ }^{T}}\right)^{\dagger} \boldsymbol{y}_{\mathrm{per}}^{-}=\mathcal{K} \mathcal{E}^{-}+o(1) \mathcal{Y}_{\mathrm{per}}^{-}
$$

Since the signal has bounded moments of order 4 , it holds $o(1) \mathcal{Y}_{\text {per }}^{-}=o(1)$.

2. Proof of $\mathcal{E}_{\mathcal{K}}^{-} \mathcal{X}_{\mathrm{per}}^{-T} \rightarrow 0$

With (28) it holds

$$
\mathcal{E}_{\mathcal{K}}^{-} \mathcal{X}_{\text {per }}^{-T}=\frac{1}{N} \sum_{l=0}^{N-1}\left(\mathcal{K}_{l}-\mathcal{K}\right)\left[\begin{array}{c}
e_{l} \\
e_{l+1} \\
\vdots \\
e_{l+p-1}
\end{array}\right] \hat{x}_{l}^{\text {per } T}=\frac{1}{N} \sum_{l=0}^{N-1} \sum_{j=0}^{p-1}\left[\mathcal{K}_{l}-\mathcal{K}\right]_{j} e_{l+j} \hat{x}_{l}^{\text {per } T}
$$

where $\left[\mathcal{K}_{l}-\mathcal{K}\right]_{j}$ indicates the $(j+1)$-th block column of matrix $\left(\mathcal{K}_{l}-\mathcal{K}\right)$. Recall that the Kalman gain is deterministic and independent of the observations. It converges linearly in the periodic part [33] and exponentially for the other components [37], thus $\left\|\mathcal{K}_{l}-\mathcal{K}\right\|=O(1 / l)$.

Denote $\tilde{e}_{l, j}=\left[\mathcal{K}_{l}-\mathcal{K}\right]_{j} e_{l+j}$. Since the innovations have bounded fourth order moments, this is also the case for $\tilde{e}_{l, j}$. Analogously to part 1 of the proof, it follows with [36] that $\frac{1}{N} \sum_{l} \tilde{e}_{l, j} \hat{x}_{l}^{\text {per } T}=o(1)$ for $j \geq 0$, finally $\mathcal{E}_{\mathcal{K}}^{-} \mathcal{X}_{\text {per }}^{-T}=o(1)$ and thus $\mathcal{E}_{\mathcal{K}}^{-} \mathcal{Y}_{\mathrm{per}}^{-T}=\mathcal{E}_{\mathcal{K}}^{-} \mathcal{X}_{\mathrm{per}}^{-T} \Gamma_{\mathrm{per}}{ }^{T}=o(1)$. Thus the fourth term in (C.1) yields

$$
\mathcal{E}_{\mathcal{K}}^{-} / \mathcal{Y}_{\text {per }}^{-}{ }^{\perp}=\mathcal{E}_{\mathcal{K}}^{-}-\mathcal{E}_{\mathcal{K}}^{-} \mathcal{Y}_{\text {per }}^{-T}\left(\mathcal{Y}_{\text {per }}^{-} \mathcal{Y}_{\text {per }}^{-T}\right)^{\dagger} \mathcal{Y}_{\text {per }}^{-}=\mathcal{E}_{\mathcal{K}}^{-}+o(1) \mathcal{Y}_{\text {per }}^{-}
$$

\section{Proof of $\mathcal{X}_{\mathrm{sys}}^{-} \mathcal{X}_{\mathrm{per}}^{-T} \rightarrow 0$}

The columns of matrices $\mathcal{X}_{\mathrm{sys}}^{-}$and $\mathcal{X}_{\mathrm{per}}^{-}$are the components of the Kalman states $\hat{x}_{k}^{V}$ corresponding to the system and periodic parts, respectively, in the real-valued modal basis after similarity transform (21) with $V$ defined in (22). Denote these parts by $\hat{x}_{k}^{V \text {,sys }}$ and $\hat{x}_{k}^{V, \text { per }}$, respectively.

For simplicity of notation we carry out the proof in the complex-valued modal basis defined by $V_{c}=\left[\begin{array}{ll}\Psi & \bar{\Psi}\end{array}\right]$ (cf. (22)), thus $A^{V_{c}}=V_{c}^{-1} A V_{c}=\operatorname{diag}(\Lambda, \bar{\Lambda})$. Then, the system and periodic parts of the states $\hat{x}_{k}^{V_{c}}=V_{c}^{-1} \hat{x}_{k}$ relate to $\hat{x}_{k}^{V, \text { sys }}$ and $\hat{x}_{k}^{V, \text { per }}$ by

$$
\hat{x}_{k}^{V, \text { sys }}=T_{m}^{-1} \hat{x}_{k}^{V_{c}, \text { sys }}, \quad \hat{x}_{k}^{V, \text { per }}=T_{h}^{-1} \hat{x}_{k}^{V_{c}, \text { per }}, \quad T_{a}=\frac{1}{2}\left[\begin{array}{cc}
\mathcal{I}_{a} & -\mathrm{i} \mathcal{I}_{a} \\
\mathcal{I}_{a} & \mathrm{i} \mathcal{I}_{a}
\end{array}\right],
$$

where $m$ and $h$ are the number of system and periodic mode pairs (see Section 2.2). Denote the $i$-th component

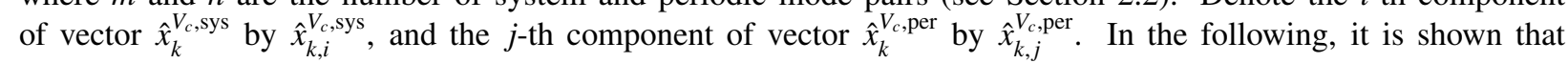
$\frac{1}{N} \sum_{k} \hat{x}_{k, i}^{V_{c} \text {,sys }} \hat{x}_{k, j}^{V_{c}, \text { per }} \rightarrow 0$ for any $i, j$, from where $\mathcal{X}_{\text {sys }}^{-} \mathcal{X}_{\mathrm{per}}^{-T} \rightarrow 0$ follows with (C.5).

From the state equation it follows

$$
\begin{aligned}
\frac{1}{N} \sum_{k=0}^{N-1} \hat{x}_{k+1, i}^{V_{c}, \text { sys }} \hat{x}_{k+1, j}^{V_{c} \text {,per }} & =\frac{1}{N} \sum_{k=0}^{N-1}\left(\lambda_{i}^{\text {sys }} \hat{x}_{k, i}^{V_{c}, \text { sys }}+K_{k, i}^{V_{c} \text {,sys }} e_{k}\right)\left(\lambda_{j}^{\text {per }} \hat{x}_{k, j}^{V_{c}, \text { per }}+K_{k, j}^{V_{c}, \text { per }} e_{k}\right) \\
& =\frac{1}{N} \sum_{k=0}^{N-1}\left(\lambda_{i}^{\text {sys }} \lambda_{j}^{\text {per }} \hat{x}_{k, i}^{V_{c} \text { sys }} \hat{x}_{k, j}^{V_{c}, \text { per }}+\lambda_{i}^{\text {sys }} \hat{x}_{k, i}^{V_{c}, \text { sys }} e_{k}^{T} K_{k, j}^{V_{c}, \text { per } T}+\lambda_{j}^{\text {per }} \hat{x}_{k, j}^{V_{c}, \text { per }} e_{k}^{T} K_{k, i}^{V_{c}, \text { sys }}{ }^{T}+K_{k, i}^{V_{c}, \text { sys }} e_{k} e_{k}^{T} K_{k, j}^{V_{c}, \text { per } T}\right)
\end{aligned}
$$

where $K_{k, i}^{V_{c} \text {,sys }}$ is the $i$-th row of the system part of the Kalman gain $K_{k}^{V_{c}}$, and $K_{k, j}^{V_{c} \text {,per }}$ is the $j$-th row of its periodic part. Then

$$
\begin{aligned}
& \left(1-\lambda_{i}^{\mathrm{sys}} \lambda_{j}^{\mathrm{per}}\right) \frac{1}{N} \sum_{k=0}^{N-1} \hat{x}_{k, i}^{V_{c}, \text { sys }} \hat{x}_{k, j}^{V_{c}, \text { per }}+\frac{1}{N} \hat{x}_{0, i}^{V_{c}, \text { sys }} \hat{x}_{0, j}^{V_{c}, \text { per }}-\frac{1}{N} \hat{x}_{N, i}^{V_{c}, \text { sys }} \hat{x}_{N, j}^{V_{c}, \text { per }} \\
& =\frac{1}{N} \sum_{k=0}^{N-1} \lambda_{i}^{\text {sys }} \hat{x}_{k, i}^{V_{c} \text {,sys }} e_{k}^{T} K_{k, j}^{V_{c}, \text { per }}{ }^{T}+\frac{1}{N} \sum_{k=0}^{N-1} \lambda_{j}^{\text {per }} \hat{x}_{k, j}^{V_{c}, \text { per }} e_{k}^{T} K_{k, i}^{V_{c}, \text { sys }}{ }^{T}+\frac{1}{N} \sum_{k=0}^{N-1} K_{k, i}^{V_{c}, \text { sys }} e_{k} e_{k}^{T} K_{k, j}^{V_{c}, \text { per } T} .
\end{aligned}
$$


The first term on the right hand side goes to zero since $\hat{x}_{k}^{\text {sys }}$ and $e_{k}$ are independent. The second term goes to zero in a similar manner. The third term is a mean of the independent process $\mathcal{L}_{k}=K_{k, i}^{V_{c} \text {,sys }} e_{k} e_{k}^{T} K_{k, j}^{V_{c} \text {,per }}{ }^{T}$ since $e_{k}$ is an independent Gaussian process. Let $v_{k}=\mathrm{E}\left(\mathcal{L}_{k}\right)=K_{k, i}^{V_{c}, \text { sys }} Q_{k} K_{k, j}^{V_{c}, \text { per }}{ }^{T}$, where $Q_{k}$ is the innovation covariance at $k$ that is bounded since the fourth moment of the innovation is bounded. Thus, it holds $\sum \mathrm{E}\left(\mathcal{L}_{k}-v_{k}\right)^{2} / k^{2}<\infty$. Then [38, Theorem 3.7] (or [39, Theorem 5.4.1]) can be applied, yielding $\frac{1}{N} \sum \mathcal{L}_{k}-\frac{1}{N} \sum v_{k}=o(1)$, and since $\left\|\frac{1}{N} \sum v_{k}\right\| \leq$ $\frac{1}{N} \sum\left\|K_{k, i}^{V_{c} \text {,sys }}\right\|\left\|Q_{k}\right\|\left\|K_{k, j}^{V_{c} \text {,per }}\right\| \leq \sup _{k}\left\|Q_{k}\right\| \frac{O(1)}{N} \sum_{k=0}^{N-1} O(1 / k)=o(1)$, the third term on the right hand side of (C.6) goes to zero. The remaining terms on the left hand side yield $\frac{1}{N} \hat{x}_{0, i}^{V_{c} \text {,sys }} \hat{x}_{0, j}^{V_{c} \text {,per }}-\frac{1}{N} \hat{x}_{N, i}^{V_{c} \text {,sys }} \hat{x}_{N, j}^{V_{c} \text {,per }} \rightarrow 0$ by the Chebyshev inequality and Borel Cantelli Lemma, since the moments are bounded. Finally, $\mathcal{X}_{\text {sys }}^{-} \mathcal{X}_{\text {per }}^{-T}=o(1)$ and

$$
\mathcal{X}_{\mathrm{sys}}^{-} / \mathcal{Y}_{\mathrm{per}}^{-}{ }^{\perp}=\mathcal{X}_{\mathrm{sys}}^{-}+o(1) \mathcal{Y}_{\mathrm{per}}^{-} \text {. }
$$

\section{End of the proof}

Plugging (C.3), (C.4) and (C.7) into (C.1) yields

$$
\boldsymbol{Y}_{\mathrm{pro}}^{-}=\Gamma_{\mathrm{sys}} \mathcal{X}_{\mathrm{sys}}^{-}+\mathcal{K} \mathcal{E}^{-}+\mathcal{E}_{\mathcal{K}}^{-}+o(1) \mathcal{Y}_{\mathrm{per}}^{-}
$$

Analogously, the relation for $\mathcal{Y}_{\text {pro }}^{+}=\mathcal{Y}_{\text {raw }}^{+} / \mathcal{Y}_{\text {per }}^{+}{ }^{\perp}$ is obtained as

$$
\mathcal{Y}_{\text {pro }}^{+}=\Gamma_{\text {sys }} \mathcal{X}_{\text {sys }}^{+}+\mathcal{K} \mathcal{E}^{+}+\mathcal{E}_{\mathcal{K}}^{+}+o(1) \mathcal{Y}_{\text {per }}^{+},
$$

To conclude the proof, since $\mathcal{Y}_{\text {per }}^{-} \mathcal{Y}_{\text {per }}^{-T}$ and $\mathcal{Y}_{\text {per }}^{+} \mathcal{Y}_{\text {per }}^{+T}$ are bounded, the norm of the remainder term is $o(1)$.

\section{Appendix D. Proof of Theorem 4}

Since $\mathcal{Y}_{\text {pro }}^{-}=\mathcal{Y}_{\text {raw }}^{-}-\mathcal{Y}_{\text {raw }}^{-} / \mathcal{Y}_{\text {per }}^{-}$, the projection in (31) yields

$$
\begin{aligned}
& \mathcal{H}=\mathcal{Y}_{\text {pro }}^{+} / \mathcal{Y}_{\text {pro }}^{-}=\mathcal{Y}_{\text {pro }}^{+}\left(\mathcal{Y}_{\text {raw }}^{-}-\mathcal{Y}_{\text {raw }}^{-} / \mathcal{Y}_{\text {per }}^{-}\right)^{T} \underbrace{\left(\mathcal{Y}_{\text {pro }}^{-} \mathcal{Y}_{\text {pro }}^{-}\right)^{\dagger} \mathcal{Y}_{\text {pro }}^{-}}_{=\mathcal{W}_{1}} \\
& =(\underbrace{\mathcal{y}_{\text {pro }}^{+} \mathcal{Y}_{\text {raw }}^{-} T}_{=\mathcal{P}_{1}}-\underbrace{\mathcal{y}_{\text {pro }}^{+} \mathcal{Y}_{\text {per }}^{-T}}_{=\mathcal{P}_{2}} \underbrace{\left(y_{\text {per }}^{-} \mathcal{Y}_{\text {per }}^{-T}\right)^{\dagger} \mathcal{Y}_{\text {per }}^{-} \mathcal{Y}_{\text {raw }}^{-} T}_{\mathcal{W}_{2}}) \mathcal{W}_{1}
\end{aligned}
$$

\section{Preliminaries}

The components of $\mathcal{Y}_{\text {raw }}^{-}$are observations of the considered linear system. Thus, the moments of $\mathcal{Y}_{\text {raw }}^{-}$derive from the moments of the underlying state and innovations. Analogously to (C.2), it holds $\mathrm{E}\left(e_{k} y_{k-j}^{T}\right)=0 \forall j>0$, since $\mathrm{E}\left(e_{k} y_{k-j}^{T}\right)=\mathrm{E}\left(\mathrm{E}\left(e_{k} \mid \mathcal{F}_{k-1}\right) y_{k-j}^{T}\right)=0$ and it can be proved that $\mathcal{E}^{+} \mathcal{Y}_{\text {raw }}^{-}{ }^{T} \rightarrow 0$ and $\mathcal{E}_{\mathcal{K}}^{+} \mathcal{Y}_{\text {raw }}^{-}{ }^{T} \rightarrow 0$ similarly to Appendix C.

\section{Asymptotic formulations for $\mathcal{P}_{1}$ and $\mathcal{P}_{2}$}

Plugging (C.9) into $\mathcal{P}_{1}$ and $\mathcal{P}_{2}$ yields

$$
\begin{aligned}
& \mathcal{P}_{1}=\Gamma_{\text {sys }} \mathcal{X}_{\text {sys }}^{+} \mathcal{Y}_{\text {raw }}^{-{ }^{T}}+\mathcal{K} \mathcal{E}^{+} \mathcal{Y}_{\text {raw }}^{-T}+\mathcal{E}_{\mathcal{K}}^{+} \mathcal{Y}_{\text {raw }}^{-T}+o(1) \mathcal{Y}_{\text {per }}^{+} \mathcal{Y}_{\text {raw }}^{-T} \\
& \mathcal{P}_{2}=\Gamma_{\text {sys }} \mathcal{X}_{\text {sys }}^{+} \mathcal{Y}_{\text {per }}^{-T}+\mathcal{K} \mathcal{E}^{+} \mathcal{Y}_{\text {per }}^{-T}+\mathcal{E}_{\mathcal{K}}^{+} \mathcal{Y}_{\text {per }}^{-T}+o(1) \mathcal{Y}_{\text {per }}^{+} \mathcal{Y}_{\text {per }}^{-T}
\end{aligned}
$$

The matrix $\mathcal{Y}_{\text {per }}^{+} \mathcal{Y}_{\text {per }}^{-}{ }^{T}$ is bounded since it is filled by covariances of the signal $\boldsymbol{Y}_{\text {per }}$, which are bounded since the signal has bounded moments of order 4. Thus $o(1) \mathcal{y}_{\text {per }}^{+} \mathcal{Y}_{\text {per }}^{-{ }^{T}}=o(1)$. The matrix $\boldsymbol{Y}_{\text {per }}^{+} \boldsymbol{y}_{\text {raw }}^{-}{ }^{T}$ is bounded too, since a Cauchy Schwartz inequality relates $\mathcal{Y}_{\text {per }}^{+} \mathcal{Y}_{\text {raw }}^{-{ }^{T}}$ to $\mathcal{Y}_{\text {per }}^{+} \mathcal{y}_{\text {per }}^{+T}$ and $\mathcal{Y}_{\text {raw }}^{-} \mathcal{Y}_{\text {raw }}^{-}{ }^{T}$ which are both covariance matrices and thus bounded because the signals $\mathcal{Y}_{\text {per }}$ and $\mathcal{Y}_{\text {raw }}$ have moments of order 4 . Recall that $\mathcal{E}^{+} \mathcal{Y}_{\text {per }}^{-T} \rightarrow 0$ and $\mathcal{E}_{\mathcal{K}}^{+} \mathcal{Y}_{\text {per }}^{-T} \rightarrow 0$, and that $\mathcal{E}^{+} \mathcal{Y}_{\text {raw }}^{-}{ }^{T} \rightarrow 0$ and $\mathcal{E}_{\mathcal{K}}^{+} \mathcal{Y}_{\text {raw }}^{-}{ }^{T} \rightarrow 0$. Then, $\mathcal{P}_{1}$ and $\mathcal{P}_{2}$ simplify to

$$
\mathcal{P}_{1}=\Gamma_{\text {sys }} \mathcal{X}_{\text {sys }}^{+} \mathcal{Y}_{\text {raw }}^{-{ }^{T}}+o(1), \quad \mathcal{P}_{2}=\Gamma_{\text {sys }} \mathcal{X}_{\text {sys }}^{+} \mathcal{Y}_{\text {per }}^{-T}+o(1) .
$$




\section{Asymptotic expression of the UPC projection}

Subsequently the projection in (D.1) yields

$$
\mathcal{H}=\mathcal{Y}_{\text {pro }}^{+} / \mathcal{Y}_{\text {pro }}^{-}=\Gamma_{\text {sys }} \mathcal{X}_{\text {sys }}^{+}\left(\mathcal{Y}_{\text {raw }}^{-}{ }^{T}-\mathcal{Y}_{\text {per }}^{-T} \mathcal{W}_{2}\right) \mathcal{W}_{1}+o(1) W_{1}+o(1) W_{2} \mathcal{W}_{1}
$$

Matrix $\mathcal{W}_{2}$ is the product of bounded matrix $\left(\mathcal{Y}_{\text {per }}^{-} \mathcal{Y}_{\text {per }}^{-T}\right)^{\dagger}$ (see Appendix C, part 1) and $\mathcal{Y}_{\text {per }}^{-} \mathcal{Y}_{\text {raw }}^{-}{ }^{T}$, which can also be proved to be bounded similarly to $\mathcal{Y}_{\text {per }}^{+} \mathcal{Y}_{\text {raw }}^{-}{ }^{T}$. Next we show that $\mathcal{Y}_{\text {pro }}^{-} \mathcal{Y}_{\text {pro }}^{-T}$ is of full rank. With (C.8) it follows

$$
\mathcal{Y}_{\text {pro }}^{-} \mathcal{Y}_{\text {pro }}^{-T}=\Gamma_{\text {sys }} \mathcal{X}_{\text {sys }}^{-} \mathcal{X}_{\text {sys }}^{-T} \Gamma_{\text {sys }}^{T}+\mathcal{K} \mathcal{E}^{-} \mathcal{E}^{-T} \mathcal{K}^{T}+\mathcal{E}_{\mathcal{K}}^{-} \mathcal{E}_{\mathcal{K}}^{-T}+\mathcal{L}+\mathcal{L}^{T}+(\text { cross terms }) \times o(1)
$$

where $\mathcal{L}=\Gamma_{\text {sys }} \mathcal{X}_{\text {sys }}^{-} \mathcal{E}^{-T} \mathcal{K}^{T}+\Gamma_{\text {sys }} \mathcal{X}_{\text {sys }}^{-} \mathcal{E}_{\mathcal{K}}^{-T}+\mathcal{K} \mathcal{E}^{-} \mathcal{E}_{\mathcal{K}}^{-T}$. The term $\mathcal{E}_{\mathcal{K}}^{-} \mathcal{E}_{\mathcal{K}}^{-T}$ in (D.2) is the diagonal covariance of $\mathcal{E}_{\mathcal{K}}^{-}$, whose norm is decreasing as the gain converges, so this goes to zero. Thus, the third term of $\mathcal{L}$ also goes to zero by Cauchy Schartz inequality. The first two terms of $\mathcal{L}$ are $o(1)$ analogously to Appendix C. All the cross terms in (D.2) are bounded, hence

$$
\mathcal{Y}_{\text {pro }}^{-} \mathcal{Y}_{\text {pro }}^{-T}=\Gamma_{\text {sys }} \mathcal{X}_{\text {sys }}^{-} \mathcal{X}_{\text {sys }}^{-T} \Gamma_{\text {sys }}^{T}+\mathcal{K} \mathcal{E}^{-} \mathcal{E}^{-T} \mathcal{K}^{T}+o(1)
$$

The first matrix is positive semi-definite, and since $\mathcal{E}^{-} \mathcal{E}^{-T}$ is positive definite and $\mathcal{K}$ is of full rank, the smallest singular value of matrix $\mathcal{Y}_{\text {pro }}^{-} \mathcal{Y}_{\text {pro }}^{-T}$ is bounded from below for $N$ large enough. Thus $\left(\mathcal{Y}_{\mathrm{pro}}^{-} \boldsymbol{Y}_{\text {pro }}^{-T}\right)^{\dagger}$ is bounded and

$$
\mathcal{H}=\Gamma_{\text {sys }} \mathcal{X}_{\text {sys }}^{+}\left(\mathcal{Y}_{\text {raw }}^{-T}-\mathcal{Y}_{\text {per }}^{-T} \mathcal{W}_{2}\right) \mathcal{W}_{1}+o(1) \mathcal{Y}_{\text {pro }}^{-}
$$

4. Relation of UPC projection to $\mathcal{Y}_{\text {sys }}^{-}$and end of proof

Since $\boldsymbol{y}_{\text {pro }}^{-}=\mathcal{Y}_{\text {raw }}^{-}{ }^{T}-\mathcal{y}_{\text {per }}^{-T} \boldsymbol{W}_{2}$, it follows from (D.3)

$$
\mathcal{H}=\Gamma_{\text {sys }} \mathcal{X}_{\text {sys }}^{+} \mathcal{Y}_{\text {pro }}^{-T}\left(\mathcal{Y}_{\text {pro }}^{-} \mathcal{Y}_{\text {pro }}^{-T}\right)^{-1} \mathcal{Y}_{\text {pro }}^{-}+o(1) \mathcal{Y}_{\text {pro }}^{-}
$$

where $\mathcal{Y}_{\text {pro }}^{-}=\mathcal{Y}_{\text {sys }}^{-}+\mathcal{E}_{\mathcal{K}}^{-}+o(1) \mathcal{Y}_{\text {per }}^{-}$, and define $\mathcal{Y}_{\text {sys }}^{-}=\Gamma_{\text {sys }} \mathcal{X}_{\text {sys }}^{-}+\mathcal{K} \mathcal{E}^{-}$. Then $\left(\mathcal{Y}_{\text {pro }}^{-} \mathcal{Y}_{\text {pro }}^{-}\right)^{\dagger}=\left(\mathcal{Y}_{\text {sys }}^{-} \mathcal{Y}_{\text {sys }}^{-T}+o(1)\right)^{-1}=$ $\left(\mathcal{Y}_{\text {sys }}^{-} \mathcal{Y}_{\text {sys }}^{-T}\right)^{-1}+o(1)$ by the matrix inverse sensitivity, and the bounds proved previously. Finally,

$$
\begin{aligned}
\mathcal{X}_{\mathrm{sys}}^{+} \mathcal{Y}_{\mathrm{pro}}^{-T} & =\mathcal{X}_{\mathrm{sys}}^{+}\left(\mathcal{Y}_{\mathrm{sys}}^{-}+\mathcal{E}_{\mathcal{K}}^{-}+o(1) \mathcal{Y}_{\mathrm{per}}^{-}\right)^{T} \\
& =\mathcal{X}_{\mathrm{sys}}^{+} \mathcal{Y}_{\mathrm{sys}}^{-T}+\mathcal{X}_{\mathrm{sys}}^{+} \mathcal{E}_{\mathcal{K}}^{-T}+\mathcal{X}_{\mathrm{sys}}^{+} \mathcal{Y}_{\mathrm{per}}^{-T} o(1)=\mathcal{X}_{\mathrm{sys}}^{+} \mathcal{Y}_{\mathrm{sys}}^{-T}+o(1)
\end{aligned}
$$

and thus

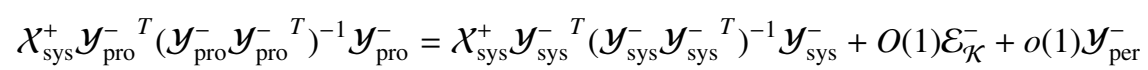

where $\mathcal{E}_{\mathcal{K}}^{-} \mathcal{E}_{\mathcal{K}}^{-T}=o(1)$. Then it follows with (D.4), since $\mathcal{E}_{\mathcal{K}}^{-} \mathcal{Y}_{\text {per }}^{-T}=o(1)$ with Appendix C, and other cross-terms are bounded,

$$
\mathcal{H}=\Gamma_{\text {sys }} \mathcal{X}_{\text {sys }}^{+} / \mathcal{Y}_{\text {sys }}^{-}+o(1)=\mathcal{Y}_{\text {sys }}^{+} / \mathcal{Y}_{\text {sys }}^{-}+o(1),
$$

since $\mathcal{E}^{-} \mathcal{Y}_{\text {sys }}^{-T}=o(1)$, and the proposed method yields the same formulation as the SSI-UPC approach computed on the so-called virtual structural system, which concludes the proof.

\section{Appendix E. Proof of Corollary 5}

The main challenge in the asymptotic study of $\mathcal{H}$ - and thus the consistency of $A$ and $C$ that are estimated from its column space - is the fact that the number of columns of $\mathcal{H}$ grows with the number of samples $N$. By evaluating the asymptotic properties of the "square" matrix $\mathcal{H} \mathcal{H}^{T}$, which has the same column space as $\mathcal{H}$ but whose dimensions are finite and non-increasing, the consistency of $A$ and $C$ can be analyzed $[16,20]$. 
Define $\mathcal{H}_{1}=\Gamma_{\text {sys }} \mathcal{X}_{\text {sys }}^{+} / \mathcal{Y}_{\text {sys }}^{-}$. Then it follows from (D.5)

$$
\begin{aligned}
\mathcal{H} \mathcal{H}^{T} & =\left(\mathcal{H}_{1}+o(1)\right)\left(\mathcal{H}_{1}+o(1)\right)^{T}=\mathcal{H}_{1} \mathcal{H}_{1}^{T}+\left(\mathcal{H}_{1}+o(1)\right) o(1)^{T}+o(1) \mathcal{H}_{1}^{T} \\
& =\mathcal{H}_{1} \mathcal{H}_{1}^{T}+o(1)
\end{aligned}
$$

since it can be proved that $\left\|\mathcal{H}_{1}\right\|$ is bounded similarly to Appendix D. In consequence, it holds

$$
\mathcal{H} \mathcal{H}^{T}=\Gamma_{\text {sys }} \mathcal{X}_{\text {sys }}^{+} / \mathcal{Y}_{\text {sys }}^{-}\left(\Gamma_{\text {sys }} \mathcal{X}_{\text {sys }}^{+} / \mathcal{Y}_{\text {sys }}^{-}\right)^{T}+o(1)
$$

This matrix satisfies Condition 1 of [16], and its consistency results from [16], Section 4.A, where the assumptions of Theorem 1 in [16] have been verified. It follows that the estimates of $(A, C)$ from $\mathcal{H}^{T}$ are consistent. Since the estimates of $(A, C)$ from $\mathcal{H} \mathcal{H}^{T}$ and $\mathcal{H}$ coincide [20], the estimates of $(A, C)$ from $\mathcal{H}$ are consistent too. This finishes the proof.

\section{References}

[1] E. Reynders, System identification methods for (operational) modal analysis: review and comparison, Archives of Computational Methods in Engineering 19 (1) (2012) 51-124.

[2] W. Weijtjens, J. Lataire, C. Devriendt, P. Guillaume, Dealing with periodical loads and harmonics in operational modal analysis using timevarying transmissibility functions, Mechanical Systems and Signal Processing 49 (1) (2014) 154 - 164.

[3] P. Mohanty, D. Rixen, Operational modal analysis in the presence of harmonic excitation, Journal of Sound and Vibration 270 (1) (2004) 93 $-109$.

[4] B. Peeters, B. Cornelis, K. Janssens, H. Van der Auweraer, Removing disturbing harmonics in operational modal analysis, in: Proceedings of the 2nd International Operational Modal Analysis Conference (IOMAC), 2007.

[5] F. Combet, L. Gelman, An automated methodology for performing time synchronous averaging of a gearbox signal without speed sensor, Mechanical Systems and Signal Processing 21 (6) (2007) 2590 - 2606.

[6] R. Randall, W. Smith, New cepstral techniques for operational modal analysis, in: Proceedings of the First World Congress on Condition Monitoring (WCCM), 2017.

[7] R. Randall, J. Antoni, W. Smith, A survey of the application of the cepstrum to structural modal analysis, Mechanical Systems and Signal Processing 118 (2019) $716-741$.

[8] J. Bienert, P. Andersen, R. Aguirre, A harmonic peak reduction technique for operational modal analysis of rotating machinery, in: Proceedings of the 1st International Operational Modal Analysis Conference (IOMAC), 2015.

[9] R. Pintelon, B. Peeters, P. Guillaume, Continuous-time operational modal analysis in the presence of harmonic disturbances-the multivariate case, Mechanical Systems and Signal Processing 24 (1) (2010) $90-105$.

[10] C. Devriendt, G. D. Sitter, S. Vanlanduit, P. Guillaume, Operational modal analysis in the presence of harmonic excitations by the use of transmissibility measurements, Mechanical Systems and Signal Processing 23 (3) (2009) $621-635$.

[11] W. Weijtjens, G. D. Sitter, C. Devriendt, P. Guillaume, Operational modal parameter estimation of MIMO systems using transmissibility functions, Automatica 50 (2) (2014) $559-564$.

[12] C. Devriendt, P. Guillaume, The use of transmissibility measurements in output-only modal analysis, Mechanical Systems and Signal Processing 21 (7) (2007) $2689-2696$.

[13] A. Maamar, M. Abdelghani, T.-P. Le, V. Gagnol, L. Sabourin, Operational modal identification in the presence of harmonic excitation, Applied Acoustics 147 (2019) $64-71$.

[14] P. van Overschee, B. de Moor, Subspace Identification for Linear Systems, 1st Edition, Kluwer, 1996.

[15] B. Peeters, G. De Roeck, Reference-based stochastic subspace identification for output-only modal analysis, Mechanical Systems and Signal Processing 13 (6) (1999) $855-878$.

[16] A. Benveniste, L. Mevel, Nonstationary consistency of subspace methods, IEEE Transactions on Automatic Control 52 (6) (2007) 974-984.

[17] M. Döhler, L. Mevel, Fast multi-order computation of system matrices in subspace-based system identification, Control Engineering Practice 20 (9) (2012) $882-894$.

[18] E. Reynders, R. Pintelon, G. De Roeck, Uncertainty bounds on modal parameters obtained from stochastic subspace identification, Mechanical Systems and Signal Processing 22 (4) (2008) 948 - 969.

[19] M. Döhler, L. Mevel, Efficient multi-order uncertainty computation for stochastic subspace identification, Mechanical Systems and Signal Processing 38 (2) (2013) 346-366.

[20] P. Mellinger, M. Döhler, L. Mevel, Variance estimation of modal parameters from output-only and input/output subspace-based system identification, Journal of Sound and Vibration 379 (Supplement C) (2016) 1 - 27.

[21] L. Ljung, System Identification (2nd Ed.): Theory for the User, Prentice Hall PTR, Upper Saddle River, NJ, USA, 1999.

[22] T. Söderström, P. Stoica (Eds.), System Identification, Prentice-Hall, Inc., Upper Saddle River, NJ, USA, 1988.

[23] M. Favaro, G. Picci, Consistency of subspace methods for signals with almost-periodic components, Automatica 48 (3) (2012) 514-520.

[24] M. Favaro, G. Picci, A subspace algorithm for extracting periodic components from multivariable signals in colored noise, IFAC Proceedings Volumes 45 (16) (2012) 1161 - 1166, 16th IFAC Symposium on System Identification.

[25] J.-N. Juang, Applied system identification, Prentice Hall, Englewood Cliffs, NJ, USA, 1994.

[26] N.-J. Jacobsen, P. Andersen, Operational modal analysis on structures with rotating parts, in: Proceedings of the International Conference on Noise and Vibration Engineering, KU Leuven, 2008. 
[27] N.-J. Jacobsen, P. Andersen, R. Brincker, Using enhanced frequency domain decomposition as a robust technique to harmonic excitation in operational modal analysis, in: Proceedings of the 2nd International Operational Modal Analysis Conference (IOMAC), 2007.

[28] P. Andersen, R. Brincker, C. Ventura, R. Cantieni, Estimating modal parameters of civil engineering structures subject to ambient and harmonic excitation, in: Proceedings of the 7th International Conference of Experimental Vibration Analysis for Civil Engineering Structures (EVACES), Porto, Portugal, 2007.

[29] A. Agneni, G. Coppotelli, C. Grappasonni, A method for the harmonic removal in operational modal analysis of rotating blades, Mechanical Systems and Signal Processing 27 (2012) $604-618$.

[30] N.-J. Jacobsen, P. Andersen, R. Brincker, Eliminating the influence of harmonic components in operational modal analysis, in: Proceedings of the 25th International Modal Analysis Conference (IMAC), 2007.

[31] Q. Zhang, On stability of the Kalman filter for discrete time output error systems, Systems \& Control Letters 107 (2017) 84-91.

[32] E. Reynders, G. De Roeck, Reference-based combined deterministic-stochastic subspace identification for experimental and operational modal analysis, Mechanical Systems and Signal Processing 22 (3) (2008) 617 - 637.

[33] S. Chan, G. Goodwin, K. Sin, Convergence properties of the Riccati difference equation in optimal filtering of nonstabilizable systems, IEEE Transactions on Automatic Control 29 (2) (1984) 110-118.

[34] V. Le Gallo, M. Goursat, L.-O. Gonidou, Damping characterization and flight identification, in: Proceedings of the 57th International Astronautical Congress (IAC), 2006.

[35] S.-E. Rosenow, S. Uhlenbrock, G. Schlottmann, Parameter extraction of ship structures in presence of stochastic and harmonic excitations, in: Proceedings of the 2nd International Operational Modal Analysis Conference (IOMAC), 2007.

[36] P. Hall, C. C. Heyde, Martingale Limit Theory and its Application, Academic Press, New York, 1980.

[37] G. Chen, G. Chen, S.-H. Hsu, Linear Stochastic Control Systems, CRC Press, 1995.

[38] H. White, Asymptotic Theory for Econometricians, Academic Press, San Diego, 1984.

[39] K. L. Chung, A Course in Probability Theory, 3rd Edition, Academic Press, San Diego, 2001. 